\title{
Heat Flow Measurement as a key to Standardization of Thermoelectric Generator Module Metrology: A Comparison of reference and absolute Techniques
}

\author{
Pawel Ziolkowski ${ }^{*}$, Przemyslaw Blaschkewitz ${ }^{a}$, Eckhard Müller $^{a, b}$ \\ ${ }^{a}$ German Aerospace Center (DLR) - Institute of Materials Research, Linder Höhe, D-51147 Cologne, Germany \\ b/nstitute of Inorganic and Analytical Chemistry, Justus Liebig University Gießen, Heinrich-Buff-Ring 17, D-35392 \\ Gießen, Germany
}

* Corresponding Author: Pawel Ziolkowski; Email: pawel.ziolkowski@dlr.de

\begin{abstract}
Traceable and standardized metrology for thermoelectric generator modules (TEM) is a mandatory element for an industrialization of thermoelectric applications. High measurement deviations $>20 \%$ have been observed in inter-laboratory tests on TEM properties. Such uncertainties are too high for scientific studies and seem insufficient to validate industrial benchmarks. Particularly, works on hightemperature TEM have to be supported by appropriate characterization techniques. This shall accelerate progress towards product releases for thermoelectric energy conversion.
\end{abstract}

In order to overcome the deficits this article suggests a generic procedure for TEM characterization. Such guideline has not been established or purposed so far and there may be other metrological approaches to conclude on performance characteristics of TEMs. The described procedure does not constitute per se a guarantee for lower uncertainties compared to alternative approaches. The objective of this work is to describe the underlying metrological procedures in detail and to link them to analytical descriptions for the derivation of the TEM properties. The procedure and given analytic expressions for particular measurands cover all functional TEM properties needed for a meaningful benchmarking.

The definition of procedures and analytical expressions for the determination of measurands enable the determination of uncertainties. This will be given in this work for the heat flow measurement, which is a mandatory part for TEM characterization and which poses certain metrological difficulties particularly at higher temperatures. Uncertainty of heat flow determination by means of a reference principle and an absolute guarded hot plate (GHP) technique will be deduced. Based on a standard reference material for the thermal conductivity full uncertainty budgets are derived for both methods between $373 \mathrm{~K}$ and $1023 \mathrm{~K}$. Best estimates of heat flow show deviations lower than $3 \%$ compared to a comparative FEM simulation. However, significant differences are found for the underlying uncertainty of heat flow. The combined uncertainty of the reference principle lies between $10 \%-13 \%$, whereas a significantly lower uncertainty between $0.2 \%-0.75 \%$ is observed for the GHP-based absolute method for heat flow determination.

Keywords: Metrology, Thermoelectric Generator, Thermoelectric Module, Standardization, Heat Flow Measurement, Absolute Method, Guarded Hot Plate

\section{Nomenclature}




\begin{tabular}{|c|c|}
\hline A-TEGMA & 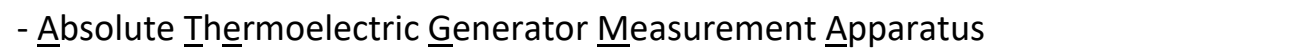 \\
\hline$c$ & - Sensitivity coefficient for uncertainty calculation \\
\hline FEM & - Finite Element Model \\
\hline GHP & - Guarded ㅂot $\underline{\text { Plate }}$ \\
\hline Guard & - Actively temperature-controlled guard heater \\
\hline$H F M$ & - Heat Flow $\underline{\text { Meter }}$ \\
\hline$\eta, \eta_{c}$ & - Efficiency of TEM, Carnot-Efficiency [\%] \\
\hline I & - Electric current $[A]$ \\
\hline$k$ & - Coverage factor for probability of uncertainty \\
\hline$K$ & - Thermal Conductance of TEM $\left[\mathrm{WK}^{-1}\right]$ \\
\hline$\kappa$ & - Thermal conductivity $\left[\mathrm{Wm}^{-1} \mathrm{~K}^{-1}\right]$ \\
\hline I & - Length of hat flow meter, which is equipped with temperature sensors [m] \\
\hline$L$ & - Length of heat flow meter $[\mathrm{m}]$ \\
\hline$N$ & - Number of thermocouples within TEM \\
\hline$P$ & - Electric power [W] \\
\hline$P I D$ & - Proportional-Integral-Derivative type of temperature controller \\
\hline$Q$ & - Heat flow [W] \\
\hline$R$ & - Electric resistance $[\Omega]$ \\
\hline REF & - Reference principle for heat flow determination \\
\hline RSS & - Rapid Steady State method for determination of internal TEM resistance \\
\hline$S$ & - Seebeck coefficient $\left[\mathrm{VK}^{-1}\right]$ \\
\hline SRM & - Standard Reference Material \\
\hline SS & 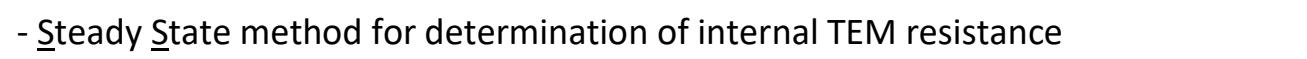 \\
\hline$T, \Delta T, \nabla T$ & - Temperature $[\mathrm{K}]$, temperature difference $[\mathrm{K}]$, temperature gradient $\left[\mathrm{Km}^{-1}\right]$ \\
\hline$T_{\mathrm{C}}, T_{\mathrm{H}}, T_{\mathrm{i}}, T_{\mathrm{m}}$ & - Cold and hot side temperature, temperature of sensor " $i$ ", mean temperature $[K]$ \\
\hline$T E$ & - Thermoelectric \\
\hline TEG & - Thermoelectric Generator \\
\hline TEGMA & - Ihermoelectric Generator $\underline{\text { Measurement } \underline{A} p \text { paratus }}$ \\
\hline TEM & - Thermoelectric Generator Module \\
\hline$u$ & - Uncertainty of particular measurand \\
\hline$V$ & - Electric voltage $[\mathrm{V}]$ \\
\hline W & - Width of heat flow meter [m] \\
\hline$x$ & - Thermal conductance between heaters of the guarded hot plate $\left[\mathrm{WK}^{-1}\right]$ \\
\hline$z_{i}$ & - Distance of temperature sensor "i" within a heat flow meter \\
\hline$Z T$ & - Figure of merit [] \\
\hline
\end{tabular}

\section{INTRODUCTION}

Thermoelectric generator (TEG) systems that can convert heat into electric energy are built from one or several thermoelectric modules (TEM). A TEM contains a variable number of $p / n$ thermocouples. The thermocouples are connected electrically in series by metallic bridges (Figure 1). A thermocouple which is operated under a temperature difference between a heat source and a heat sink generates a thermovoltage at its terminals due to the Seebeck effect [1]. 


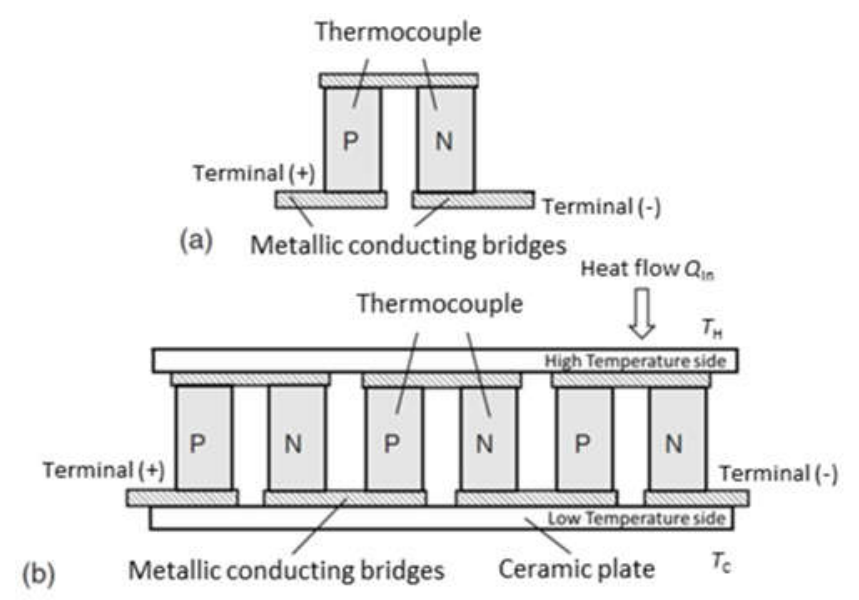

Figure 1: Schematic of a thermocouple (a), which is the basic element for a TEM (b). The TEM absorbs a heat flow $Q_{\text {In }}$ at the hot side, which operates at $T_{\mathrm{H}}$, and releases the heat at the cold temperature side, which is kept at $T_{\mathrm{C}}$. Due to the Seebeck effect a thermovoltage is generated by the TEM, which can be tapped at its terminals.

The performance of a TEM is described by its efficiency $\eta$, which is defined as the ratio between electric power output $P$ and the incident heat flow at the hot side of a TEM $Q_{\ln }$. Due to energy conservation $\eta$ equals the ratio between $P$ and the sum of $P$ and $Q_{\text {out }}$, too. $Q_{\text {out }}$ is the released heat flow at the cold side of the TEM.

$$
\eta=\frac{P}{Q_{\text {In }}}=\frac{P}{P+Q_{\text {out }}}
$$

The maximum conversion efficiency $\eta_{\mathrm{MAx}}$ is limited by the Carnot efficiency $\eta_{\mathrm{C}}=\left(T_{\mathrm{H}}-T_{\mathrm{C}}\right) / T_{\mathrm{H}}$, which is related to the hot side temperature $T_{\mathrm{H}}$ and cold side temperature $T_{\mathrm{C}}$ at the TEM. In fact, physical properties of the thermoelectric materials and loss mechanisms in the module affect $\eta_{\text {MAX }}$ by the module's figure of merit $Z T_{\text {TEM }}=S^{2} /\left(R_{\mathrm{i}} \cdot K\right) \cdot T_{\mathrm{m}}$ which is defined by the effective value of the Seebeck coefficient $S$, the thermal conductance $K$, and the electric resistance $R_{\mathrm{i}}$ of the module with respect to the temperature interval of operation and its mean temperature $T_{\mathrm{m}}=\left(T_{\mathrm{H}}-T_{\mathrm{C}}\right) / 2[2]$.

$$
\eta_{M A X}=\frac{T_{H}-T_{C}}{T_{H}} \frac{\sqrt{1+Z T_{T E M}}-1}{\sqrt{1+Z T_{T E M}}+\frac{T_{C}}{T_{H}}}
$$

Many application scenarios for TEM offer waste heat at temperatures as high as preventing the use of established $\mathrm{Bi}_{2} \mathrm{Te}_{3}$-based TEM, due to the limited temperature stability of $\mathrm{Bi}_{2} \mathrm{Te}_{3}$-based thermoelectrics. Recent developments on high temperature thermoelectric (TE) materials and contacting technologies for TEM integration resulted in the demonstration of prototypes $[3,4,5]$ and releases of small batch productions by industries $[6,7,8,9]$. Depending on the chosen thermoelectric (TE) material decent technological maturities could be shown resulting in TEM efficiencies between $8.9 \%[10]$ and $12 \%[11]$.

In view of this continuous progress the precise characterization of TEM becomes increasingly important. A successful industrialization and development of markets for TE applications in energy harvesting must be accompanied by the availability of traceable metrology with defined uncertainty budgets for module specifications.

TEM characterization techniques have to meet several requirements. On the one hand both development on contacting technologies and integration solutions and the reliable specification of 
module properties built from optimized TE materials require the validation of possibly incremental improvements and consequently the determination of measurands with high repeatability and lowest possible uncertainty. On the other hand, the experimental validation of functional stability of individual TEM prototypes or an in-line quality monitoring in industrial production put the focus on a reliable detection of relative differences to a reference value for particular measurands. This still requires information on the repeatability but is feasible with some mitigation of the uncertainty in the limits of acceptable tolerances.

Generally, measurements can be split into absolute or reference techniques. The development, operation and maintenance of absolute measurement principles usually require long methodical experience and higher expenses. Particularly for the case of absolute heat flow measurements, the required stabilization for thermal equilibrium and the complexity of the measurement systems exceed typical specifications of industrial measurement practise. This is why absolute measurement techniques are usually applied as primary methods at standardization authorities and in specific metrological and research institutions only. In contrast to primary methods, reference measurement techniques are widely applied as they can be operated easier. However, repeatability and traceability still remain mandatory for the operation of reference methods. Independently from the application case uncertainty budgets have to be specified for absolute and reference methods anyway.

Reliable specification of the TEM efficiency has remained challenging to date due to lack of guidelines and standards for testing methods. An international round robin campaign, which was accomplished among twelve laboratories revealed deviations of approximately $20 \%$ for all TEM properties. The qualification of the used comparative samples and results of this study will be published soon. In order to overcome this deficit, low uncertainty characterization methods have to be developed and qualified as primary methods. Their results build the base for the qualification of specific TEM as reference samples with low uncertainties, which are still missing up to now. Respective work on high temperature reference TEM has already been started [12] and will pave the way to set uncertainty budgets of widely used comparative measurement techniques in future.

Initially, a suggestion for a measurement protocol is given, which has proven to be an effective way for the characterization of TEM. As the measurement of heat flow turned out to be one of the most critical contributions to TEM uncertainty, an uncertainty analysis of commonly used steady-state measurement techniques is presented afterwards. Here we discuss a reference principle and introduce an absolute measurement method based on the guarded heater principle (GHP).

\section{Module characterization Techniques}

Basically, TEM characterization covers determination of electric, thermal and thermoelectric properties which have to be measured under variable installation and operation conditions. The power output, heat flow, and the efficiency of a TEM are certainly in the focus of interest. However, from the viewpoint of TEM developers and system engineers further information is needed like the open circuit voltage, optimum and short circuit current, and the electric and thermal resistance of a TEM. Several methods for determination of TEM properties have been proposed in literature. Basically all described techniques can be categorized by transient and steady state methods. Furthermore, every type can be classified whether the determination of $\eta$ is based on a measurement of electric power output and heat flow according to equation (1) or on a calculation from $Z T_{\text {TEM }}$ using equation (2). Transient methods are represented by the impedance spectroscopy [13] and Harman method [14,15]. Both techniques measure $Z T_{\text {TEM }}$ to conclude on $\eta$. Likewise McCarty 
et al. [16] and Min [17] proposed methods for the determination of $Z T_{\text {TEM, }}$ using a combination of measurands from steady state and transient conditions obtained by a switching procedure of the electric current. Mahajan et al. [18] proposed a rapid scanning of the $I-V$ characteristic of a TEM for determination of electric properties, while a guarded heater was used for heat flow measurement.

No official guideline or standard measurement protocol is available for TEMs up to now. A sequential procedure will be suggested in this section (Figure 2). The protocol is related to steady state conditions and is neither limited to a certain typology of the TEM nor to a specific temperature, pressure or current range. The described procedure does not determine technical specifications of the used device components. Users have to ensure a decent device design in order to meet with safety regulations and accepted means for low uncertainty measurements against the background of the target boundary conditions. Furthermore users have to ensure that resulting measurement conditions coincide with the model assumptions, such for instance given for the heat flow determination by means of the reference principle, which requires an one-dimensional heat flow within the applied heat flow meter. The described process involves heat flow measurements by means of reference or absolute characterization techniques. This approach is considered to provide a more meaningful interpretation of the TEM performance compared to the use of $Z T_{\text {TEM }}$ and a following evaluation by equation (2). This is due to the fact, that $Z T_{\text {TEM }}$ is frequently determined with a relatively low temperature difference of a few $\mathrm{K}$ only, whereas TEMs usually operate under several hundreds of $\mathrm{K}$ temperature difference. Though $Z T_{\text {TEM }}$ can be principally determined under higher temperature difference, too [19], equation (2) still represents an approximation function for $\eta$ only. It is based on a constant property model, which uses effective values of the temperature dependent material properties [20]. Alternatively a cumulative property model can be used to conclude on $\eta$, which was proposed by Kim et al. [21] and which accounts for the temperature dependency of material properties by means of a different expression for the maximum efficiency with the use of a so called engineering figure of merit. A recent analysis for 18 high-performance thermoelectric materials by Armstrong et al. [22] revealed an overestimated prediction of $\eta$ by the constant property model compared to the cumulative model, which equalled a factor of 1.16 on the average. Regardless of the chosen model for TEM efficiency a pure analytic or simulation-based determination of TEM properties on the base of experimentally determined material properties is subjected to significant errors too, due to necessitated assumptions on elusive electric and thermal coupling conditions and inherent uncertainties of the applied characterization methods. Thus, a direct determination of TEM properties under relevant boundary conditions remains still mandatory for a reliable and meaningful prediction of TEM operation characteristics.

\subsection{TEM characterization protocol}

Initially the TEM is installed in a thermal measuring section (Figure 3), which is usually located within a compartment in order to establish a defined atmospheric and thermal measurement environment. The TEM is thermally coupled to heat transfer blocks of the measuring section by means of graphite foils, thermal grease or similar in order to reduce the thermal contact resistance. After the TEM installation an axial pressure is applied along the column and the compartment is evacuated or set under atmospheric pressure with inert gas. Principally measurements can be likewise conducted under air in case of sufficient oxidation resistance of the device components and the TEM. In case of an atmospheric pressure additional heat conduction by convection has to be considered from a metrological viewpoint, which might affect the uncertainty of heat flow measurement. The temperature difference along the column is controlled with a heater and a cooling plate, which build 
the outer components of the measuring section. The measurement process is organized within loops, in which boundary conditions as temperatures, the axial pressure, and atmospheric conditions are varied. For a stabilized set of these boundary conditions the electric current is varied for the electric characterization of the TEM. Since all properties of a TEM are temperature dependent, every property and its corresponding uncertainty have to be determined with respect to the given temperature conditions.

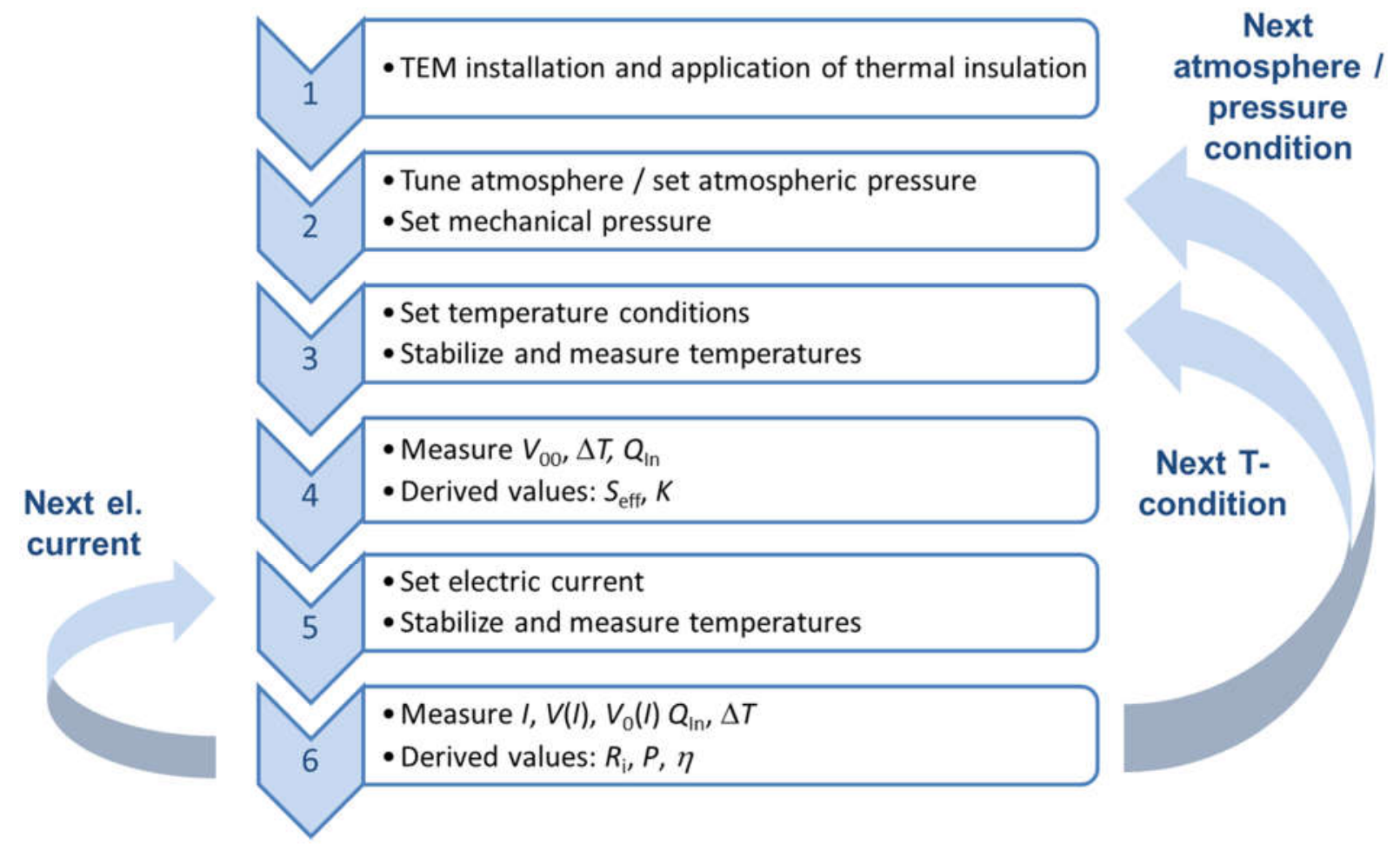

Figure 2: Generic process chart for TEM characterization involving central measurands: temperature $(T)$, open circuit voltage $\left(V_{00}\right)$, absorbed heat flow $\left(Q_{\text {In }}\right)$, temperature difference $(\Delta T)$, effective Seebeck coefficient $\left(S_{\text {eff }}\right)$, thermal conductance $(K)$, electric current $(I)$, terminal voltage $(V)$, electric resistance $\left(R_{\mathrm{i}}\right)$, electric power output $(P)$, and efficiency $(\eta)$.

The temperature difference at the TEM $\Delta T$ can be determined from the measured hot side temperature $T_{\mathrm{H}}$ and the cold side temperature $T_{\mathrm{c}}$. Both temperatures can be either sensed by a single or multiple temperature sensors placed in the direct vicinity of the TEM, or by a linear extrapolation from discretely localized temperature sensors in the direction of heat flow within the heat transfer blocks at both sides of the TEM. After temperature stabilization the open circuit voltage $V_{00}$ can be measured.

$$
V_{00}=N \cdot S_{\text {eff }} \cdot \Delta T=N \cdot S_{\text {eff }} \cdot\left(T_{\mathrm{H}}-T_{\mathrm{C}}\right)
$$

Knowing the number of installed thermocouples within the TEM $N$ and measuring $\Delta T$ gives access to the effective Seebeck coefficient $S_{\text {eff }}$ of the TEM. 


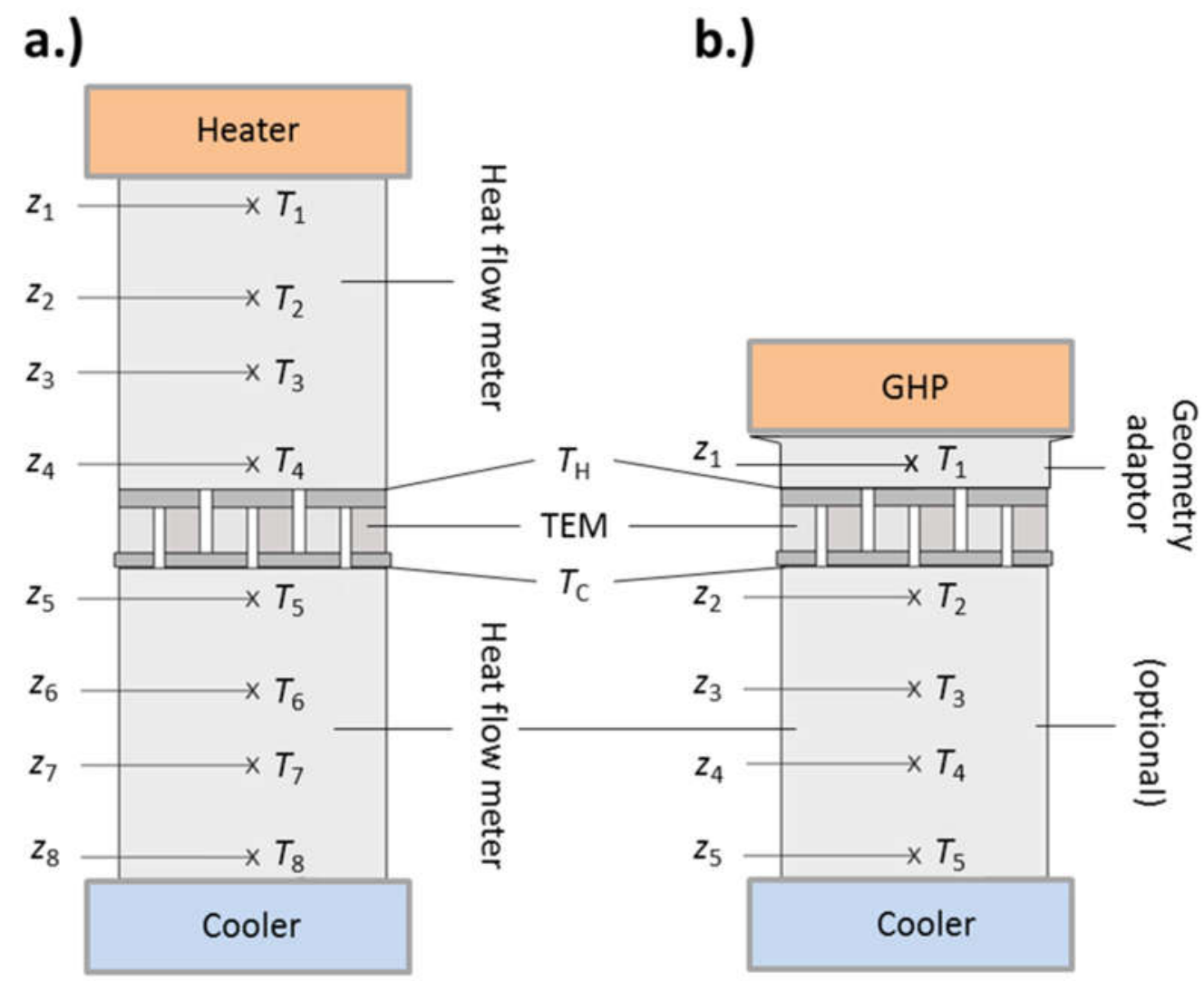

Figure 3: Schematic of measuring sections for a reference principle (a) and an absolute method (b) of heat flow measurement. The TEM (here as a symbolic representation for any kind of planar TEM) is placed thermally in series with a heater and a cooler with heat transfer blocks set in between. For the reference principle at least one heat flow meter has to be used, which contains several temperature sensors. A heat flow meter is redundant for the absolute method, which uses a guarded hot plate (GHP) as a source of a defined heat flow.

According to the one-dimensional Fourier's law the heat flow $Q$ is directly proportional to the temperature gradient $\nabla T_{\mathrm{M}}$ within the heat flow meter (HFM). Experimentally $Q$ can be determined from the temperature difference $\Delta T_{\mathrm{M}}$ along the HFM.

$$
\dot{Q}=-A_{M} \cdot \kappa_{M} \cdot \nabla T_{M}=-A_{M} \cdot \kappa_{M} \cdot \frac{\Delta T_{M}}{l_{M}}
$$

Here $\kappa_{\mathrm{M}}, A_{\mathrm{M}}$ and $I_{\mathrm{M}}$ denote the thermal conductivity, the cross section area and the length of the HFM section, which is equipped with temperature sensors. With regard to the temperature interval along the HFM, equation (4) assumes a constant $\nabla T_{M}$ and a mean value for the thermal conductivity of the HFM material. Since TEM occasionally require a heat flow of several hundred Watts, considerable temperature differences can occur along a HFM in dependence of the material choice. Consideration of the temperature dependence of $\kappa(T)$, which can cause a locally different $\nabla T_{\mathrm{M}}$, can be accomplished by a section-wise evaluation of equation (4). For the calculation of the effective heat flow every single value from a particular section has to be weighted with the respective sensor distance in case of an irregular spacing between the installed sensors.

$$
\dot{Q}=\frac{A_{M}}{\left|z_{n}-z_{1}\right|} \sum_{i=1}^{n-1}-\kappa\left(\frac{T_{i+1}+T_{i}}{2}\right) \cdot \frac{\left(T_{i+1}-T_{i}\right)}{\left(z_{i+1}-z_{i}\right)} \cdot\left|z_{i+1}-z_{i}\right|
$$

Using the reference principle the heat flow can be measured either at the hot or cold side of the TEM, or on both sides. The latter allows for a verification of the measurement uncertainty, since 
under exclusion of lateral heat losses (e.g. by radiation or convection) the energy conservation requires the sum of the cold side heat flow $Q_{\text {out }}$ and the electric power output of the TEM $P$ to be equal with the incident heat flow at the hot side of the TEM $Q_{l n}$.

Alternatively, the heat flow can be measured by means of an absolute method. Corresponding concepts are based on a guarded hot plate (GHP), which is described for instance as a standard test method for heat flux measurements by the ASTM-C177 [23]. However, the technical implementations for heat flow measurements on TEMs differ significantly from ASTM-C177, since this standard refers to measurements on monolithic and homogeneous material samples only. Some concepts and implementations for an absolute heat flow determination on TEM can be found in the literature $[24,25,26]$, which give insides into the variety of designs and instrumentations of the developed methods. The central component of this technique is a metering heater surrounded by actively controlled guard heaters, which shall prevent an uncontrolled heat loss of the GHP. The heat flow through the TEM is equated to the dissipated power of the GHP, which is determined by measurements of the electric current flow through the GHP $I_{\text {GHP }}$ and the voltage drop $V_{\mathrm{GHP}}$.

$$
\dot{Q}=V_{G H P} \cdot I_{G H P}
$$

The thermal conductance $K$ of the TEM can be defined for open circuit $(I=0)$ conditions by the ratio between $Q=Q_{\text {out }}=\left.Q_{\text {in }}\right|_{I=0}$ and $\Delta T$. It has to be noted that $K$ includes the contribution of the heat transfer coefficients between the TEM and the directly attached heat exchanging components within the measuring section.

$$
K=\frac{\dot{Q}}{\Delta T}
$$

Measurement of further TEM properties requires an electric direct current (DC) flow through the TEM. This can be accomplished either by connecting the TEM to a passive resistor network or to an electronic load. Typically the TEM current $/$ is varied from zero up to the short circuit case. Every change of current flow alters the heat flow through the TEM due to the Peltier effect [20]. Thus, after changing / additional stabilization time is required to reach thermal equilibrium again. The required time depends on the heat capacity and thermal resistance of the measuring section and can reach tens of minutes. Steady state conditions can be detected by a measurement of the residual drift of temperatures within the measuring section. Alternatively, the residual drift of the terminal voltage of a TEM can be used for the detection. In principle the terminal voltage is an excellent indicator for the stability of the effective temperature difference at the TEM. However, this method requires sufficiently high signal sensitivity and is impeded by low to moderate thermovoltages, which are generated by TEM prototypes or particularly by TEMs with a low number of thermocouples.

The power output of the TEM $P$ can be determined from concurrent measurements of $I$ and the terminal voltage $V$.

$$
P=V \cdot I
$$

The maximum power output becomes accessible from a polynomial approximation of the obtained power parabola (Figure 4a). Since the maximum power output is given for the condition of impedance matching $\left(R_{\mathrm{i}}=R_{\text {load }}\right) P_{\text {Max }}$ can be alternatively calculated from $V_{00}$ and $R_{\mathrm{i}}$.

$$
P_{\text {Max }}=\frac{V_{00}^{2}}{4 * R_{i}}
$$


$V_{00}$ denotes the open circuit voltage, which is obtained under the equilibrium temperature conditions for $T_{\mathrm{H}}$ and $T_{\mathrm{C}}$ at optimum current flow $I_{\mathrm{P} \max }$ for a maximum power output. Applying equation (1) with heat flow and power output data for every electric load current gives finally access to the efficiency of the TEM. The maximum efficiency can be determined by a polynomial approximation of the current dependent efficiency curve.

a.)

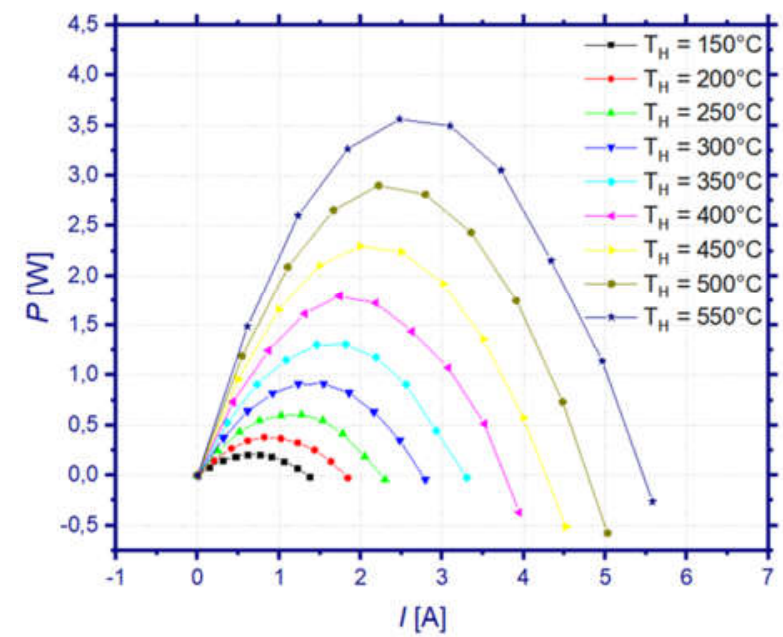

b.)

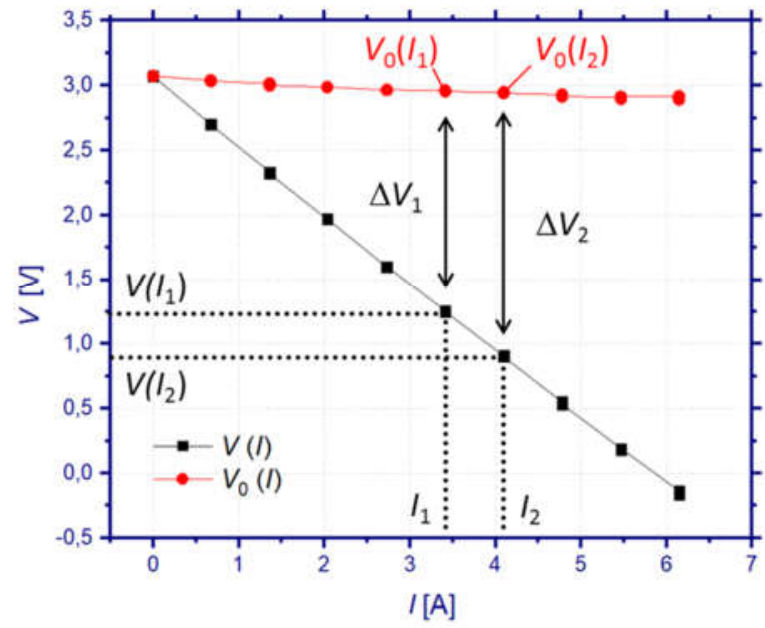

Figure 4: Measured power parabola curves (a) of a high temperature TEM, which was tested at a constant $T_{\mathrm{C}}=50{ }^{\circ} \mathrm{C}$ and various hot side temperatures $T_{\mathrm{H}}$ between $150{ }^{\circ} \mathrm{C}$ and $550{ }^{\circ} \mathrm{C}$. These temperatures relate to open circuit conditions. The measured $I-V$ curve (b) shows the TEM terminal voltage $V$ as a function of the current flow $I$ (black curve) at fixed heater and cooler temperatures. The Peltier effect causes a decreasing open circuit voltage $V_{0}(I)$ (red curve) with increasing current flow as a result of the limited thermal conductance of the heat transfer blocks in series to the TEM (see Fig. 7).

The internal electric resistance $R_{\mathrm{i}}$ can be principally obtained from the $I-V$ characteristic (black curve in Figure $4 b$ ) under steady state conditions.

$$
R_{i}^{S S}=\frac{V_{1}-V_{2}}{I_{1}-I_{2}}
$$

However, such a direct evaluation of the $I-V$ characteristic leads to an overestimation of $R_{\mathrm{i}}$. This is caused by the decreasing open circuit voltage $V_{0}(I)$ due to the Peltier effect, which lowers the effective temperature difference $\Delta T$ at the TEM in presence of a DC current flow as a result of the limited thermal conductance of the heat transfer blocks when the heater and cooler temperatures are stabilized. Thermal resistances of the TEM coupling towards the measuring section and those of ceramic plates, metallic bridges and their interfaces (within the TEM) contribute to the decrease of $V_{0}(I)$, too, since these elements lower the effective temperature difference at the TE thermocouples. A compensation of the Peltier effect on the decreasing temperature difference is possible by an adjustment of temperature set points for the heater and the cooling device in order to restore the temperature difference from open circuit conditions $(I=0)$ under current flow again. This procedure necessitates additional stabilization time to reach steady state conditions within the measuring section. However, in order to mitigate the Peltier impact on the measurement of $R_{\mathrm{i}}$ without a readjustment of the temperature difference, the decreasing level of $V_{0}(I)$ can be measured by a fast signal sampling during the switching event of $I(I \rightarrow 0)$. This technique is similar to the method, which was used by McCarty et al. [16] and Mahajan et al. [18], who has called this approach earlier as the rapid steady state (RSS) method. After thermal stabilization under a constant $l$, the TEM circuit is opened. Analog to the Harman method for direct determination of $Z T_{\text {TEM }}[14,15]$ immediately after 
the switching event the response of the terminal voltage consists of an instantaneous step function due to the ceasing Ohmic voltage drop and a transient component, which is governed by the thermal relaxation process of the effective temperature difference. A fast signal sampling and direct measurement of $V_{0}(I)$ allows for a Peltier-corrected value of $R_{\mathrm{i}}$ from measurements of $\Delta V_{1}, \Delta V_{2}$, and the corresponding current values $I_{1}$ and $I_{2}$, respectively (Figure $4 \mathrm{~b}$ ).

$$
\begin{gathered}
\Delta V_{1}=V_{0}\left(I_{1}\right)-V\left(I_{1}\right)=\left.V\right|_{I_{1} \rightarrow 0}-\left.V\right|_{I=I_{1}} \\
\Delta V_{2}=V_{0}\left(I_{2}\right)-V\left(I_{2}\right)=\left.V\right|_{I_{2} \rightarrow 0}-\left.V\right|_{I=I_{2}} \\
R_{i}^{R S S}=\frac{\Delta V_{1}-\Delta V_{2}}{I_{1}-I_{2}}
\end{gathered}
$$

Alternatively $R_{\mathrm{i}}$ can be measured likewise by application of a small alternating current (AC) signal and an evaluation according to equation (10). The choice of a sufficiently high frequency for the $A C$ current excitation avoids the impact of the Peltier effect but requires additional instrumentation for the separation of the real part and the reactance of the measured impedance, which can be accomplished by means of Lock-in amplifiers for instance.

\subsection{TEM characterization methods at DLR}

This section gives a brief survey on the custom-made characterization facilities "TEGMA" (Thermoelectric Generator Measurement Apparatus) and "A-TEGMA" (Absolute Thermoelectric Generator Measurement Apparatus), which are operated at DLR. The peripheral instrumentation of both facilities is similar to the most part, but differs only by components, which are used for thermal control and heat flow determination. The TEGMA employs a HFM according to the reference principle, whereas a GHP-based absolute method and/or a reference principle are used within the ATEGMA for heat flow measurement. The following information does not present the devices in detail unless a given relevance for the uncertainty of particular measurands, which will be discussed in later sections of this article.

a.)

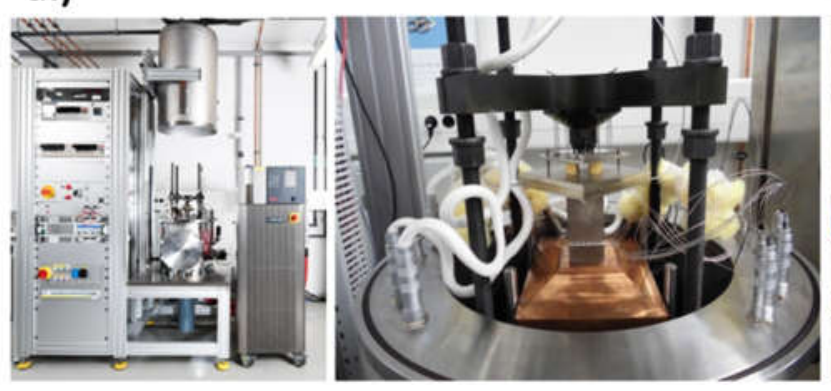

b.)

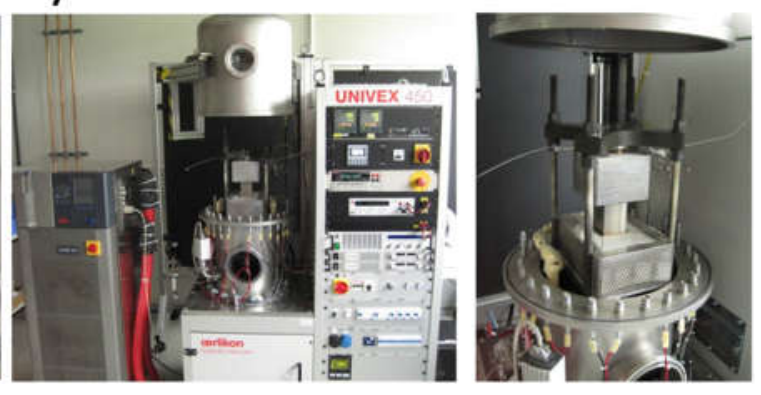

Figure 5: TEM characterization facilities "TEGMA" (a) and "A-TEGMA" (b) at DLR.

\subsubsection{Reference heat flow determination}

Both facilities use oxygen-free copper heat exchangers within their measuring sections, which improves temperature homogeneity on the coupling faces at the hot and cold side of the TEM due to the high thermal conductivity of $\mathrm{Cu}$. The use of $\mathrm{Cu}$ facilitates a homogenization of the heat flux at transitions between different components within the heat transmission path. Thus, $\mathrm{Cu}$ promotes a one-dimensional heat flow along the sensor-equipped section within the HFM, which is a mandatory precondition for a reference method of heat flow determination according to equations (4) and (5), respectively. A disadvantage of $\mathrm{Cu}$ is given for characterization of TEM under low heat flow 
conditions. Associated therewith a low $\Delta T_{\mathrm{M}}$ increases the relative uncertainty of the temperature gradient measurement for heat flow determination by means of the reference principle. After the installation of the TEM the measuring section (Figure 6) is thermally dammed in order to decrease lateral heat losses, which impair the one-dimensionality of the heat flow and consequently the uncertainty of measurements.

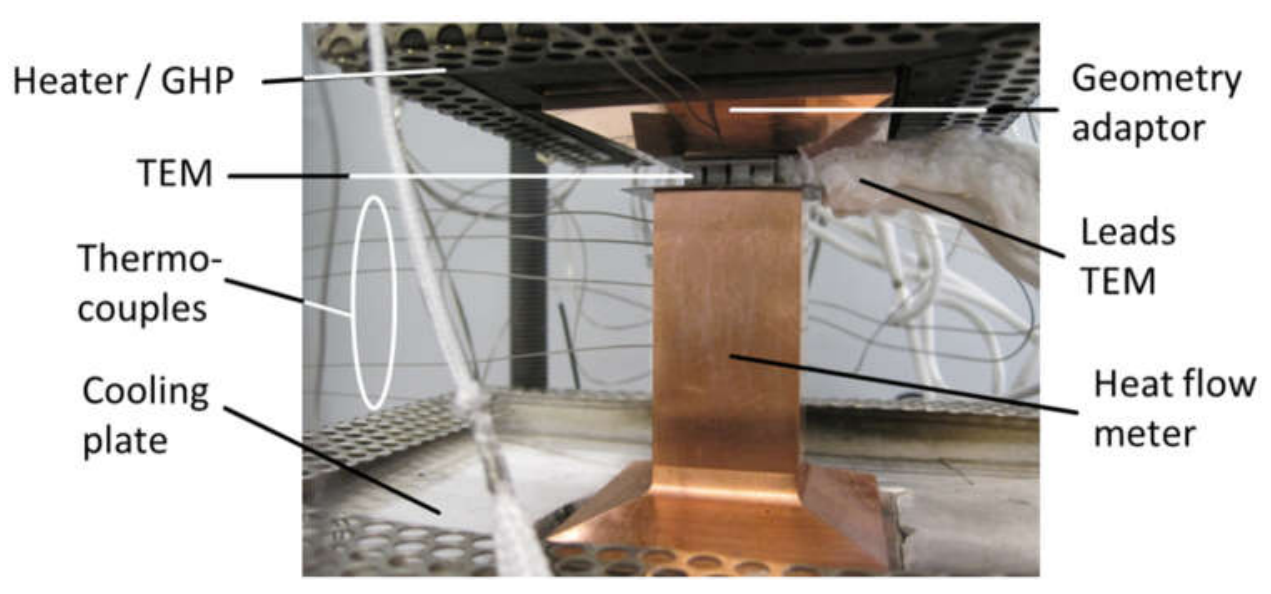

Figure 6: Measuring section of a DLR facility prior to thermal damming with HFM according to the reference principle.

Depending on the footprint of the TEM, a HFM has to be chosen with at least an equal or bigger cross section to homogenize the lateral temperature field on the coupling area of the TEM. Even though the reference principle is not constricted to heat flow measurements on the cold side, one has to consider the possible impact of radiation heat losses on the uncertainty, if measurements are conducted on the hot side of the TEM. Figure 6 shows a measuring section in which the TEM is coupled to the heater by a truncated pyramid. The pyramid is an exchangeable part for cross section adaption between the heater and the TEM. The shown part has a height of $12 \mathrm{~mm}$ and a cross section of $30 \times 30 \mathrm{~mm}$ (TEM side) and $65 \times 65 \mathrm{~mm}$ (heater side), respectively. Three type- $\mathrm{N}$ thermocouples ( $0.5 \mathrm{~mm}$ diameter, Inconel sheath, 1NI05/1000/MP/FM.N, ThermoExpert) are placed with a vertical distance of $2 \mathrm{~mm}$ within the pyramid, which allows for a determination of $T_{\mathrm{H}}$ at the hot TEM coupling face. An extrapolation technique is used within the cold side HFM for determination of

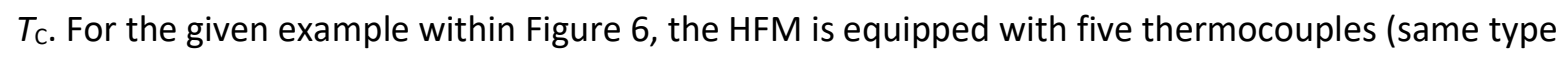
as used within the pyramid), which are placed with a gradually decreasing vertical distance between $15 \mathrm{~mm}$ and $5 \mathrm{~mm}$ in the direction towards the TEM. The sensors measure the temperature drop $\Delta T_{\mathrm{M}}$ along the cold side HFM for determination of the released heat flow at the cold side of the TEM. A $0.2 \mathrm{~mm}$ thick graphite foil is used for thermal coupling between all parts of the measuring section (heater, pyramid, TEM, heat flow meter, cooling plate).

\subsubsection{Absolute heat flow determination}

The absolute heat flow determination at DLR is accomplished by an in-house developed guarded hot plate (GHP) technique. The GHP transfers the determination of heat flow from thermal measurands to an electric measurement of the dissipated Joule heat of the metering heater. Prevention of side heat losses of the GHP is accomplished by guard heaters, which surround the GHP in order to establish isothermal conditions in every direction except towards the TEM. A thick film pyrolytic graphite heater enclosed in a boron nitride encapsulation (Boralectric ${ }^{\circledR}$, TECTRA) is used as the GHP core element, the metering heater. The GHP is embedded within a $\mathrm{Cu}$ shell, for homogenization of 
temperature and heat flow. The lower surface of the shell is used for thermal coupling with the TEM sample or with the measuring section.
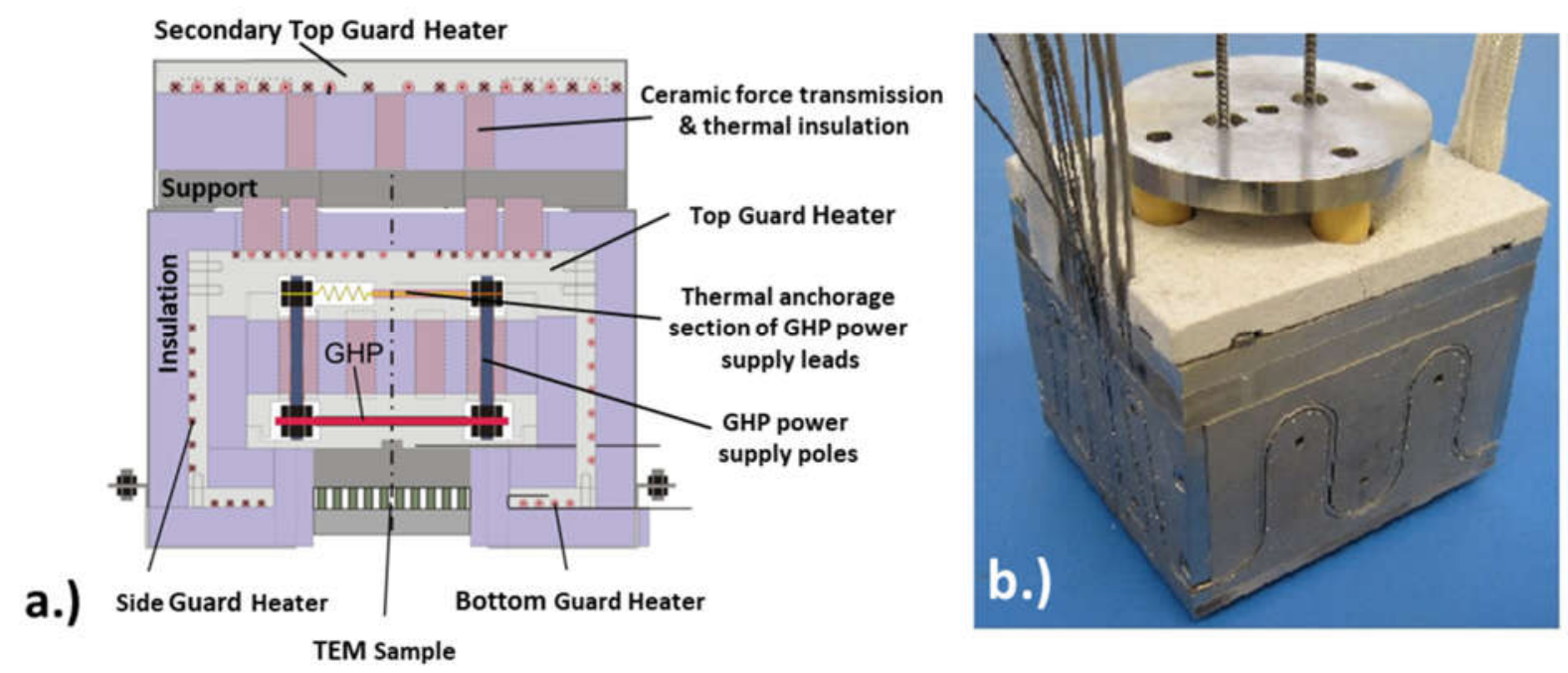

Figure 7: Schematic of the GHP-apparatus (a) showing the basic arrangement of the GHP, guard heaters, and passive insulation components and photograph of the GHP-apparatus with upper support plate, without outer insulation and secondary guard (b).

Four individually controlled guard heater circuits are used within the GHP-system for the control of the heat flow. Prevention of heat losses at the lower section of the GHP next to the coupling face of the TEM is accomplished by a bottom guard heater. During a measurement the temperature of the bottom guard heater is set to the coupling temperature of the measurement section. A side and the top guard heater are used to supress lateral heat losses of the GHP and losses towards the upper mechanical support, respectively. The temperature of the top guard heater is set to the temperature of the GHP. The setpoint of the side guard heater equals the mean temperature of the $\mathrm{Cu}$-shell, which accommodates the GHP. The electric supplying leads of the GHP can potentially act as a heat source or sink and could consequently contribute to a thermal cross talk of the GHP with its direct surrounding. Thus, the top guard heater is additionally used for thermal anchorage of these leads, which reduces the temperature difference and the thermal exchange of the GHP along these leads. TEMs are usually characterized under a variable and occasionally high axial pressure of several MPa, which has to be transmitted through the GHP-system. This introduces different heat conduction paths, which could yield a considerable heat loss of the top guard heater towards the mechanical support. This heat loss could cause an inhomogeneous temperature distribution impairing the effectiveness of thermal guarding in the upper section of the GHP. Reduction of upward heat losses of the top guard heater is accomplished by a secondary top guard heater, which is mounted above the primary one. The temperature of the secondary guard heater is set to the same setpoint as the GHP and the top guard heater. Force transmission within the GHP-system is accomplished by zirconia ceramic sleeves, which additionally supress heat exchange due to their low thermal conductivity < $2 \mathrm{Wm}^{-1} \mathrm{~K}^{-1}(\mathrm{Z}-2000$, ceratec $\mathrm{GmbH})$. Further mitigation of thermal crosstalk between the GHP and the guard heaters is accomplished by insulating material with a low thermal conductivity of $<115 \mathrm{mWm}^{-}$ ${ }^{1} \mathrm{~K}^{-1}$ (Isocal-C, TechnoPhysik Group), which is placed into the gaps of the construction. The GHPsystem is equipped with 20 type- $N$ thermocouples (same type as mentioned above) in total, which are located at different positions of the construction for temperature control of the individual heater circuits and for monitoring of temperature homogeneity. Four thermocouples are located within the lower $\mathrm{Cu}$ shell of the GHP to control its temperature and to determine the temperature of the 
sample coupling area. The side guard heater carries eight thermocouples, while the top guard heater is equipped with three temperature sensors. These sensors are located in the center of each heater plate and at off positions on the halfway towards the edges of each plate. The bottom guard carries four sensors (one for every center point of each heating plate), while the secondary guard is equipped with one sensor in the middle of the heater plate for temperature control. The heating currents of the four guard heaters and the GHP are monitored and provided by individual DC power supplies, which are controlled by two multi-channel PID controllers. Main parts on the instrumentation of both facilities are summarized within Table 1.

\subsubsection{Device Specifications}

The applied axial pressure has a direct impact on the thermal contact resistances between the TEM and the attached components of the measuring section. Related uncertainties for the measurement of heat flow and temperatures due to a pressure uncertainty are mitigated by means of a regular calibration of the installed force sensor against an in-house calibration standard. In case of an unfavourable mechanical construction of force transmitting components within the measuring section the effective pressure can even change during a measurement due to a thermal expansion of involved parts. A temperature-induced pressure change on the TEM is compensated by a free suspension of the cooling plate at the bottom of the measuring section. The axially movable cooling plate is carried by a variable set of springs, which compensate the thermal expansion of the components in the force transmission path by their compression.

Table 1: Technical instrumentation of the TEGMA and A-TEGMA facility for TEM characterization.

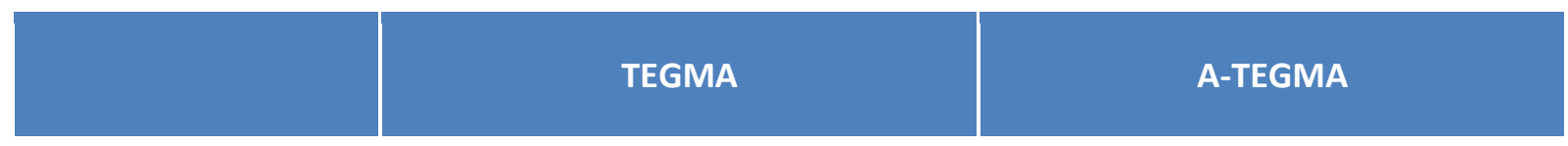

Measuring Section

Heater

Hot side heat exchanger

Heat flow meter

Cooling system

Electric Components

Heater supply

Temperature Controller

Control $I_{\mathrm{TEM}}$
Heating wire within Inconel plate

$\left(T_{\operatorname{Max}}=1273 \mathrm{~K}\right)$

Pyrolytic graphite heater as GHP $\left(T_{\operatorname{Max}}=1023 \mathrm{~K}\right)$
Cu pyramids with variable cross section for TEM coupling

Cu block@ cold side

GHP / Cu block@ cold side

Cu cooling plate connected to chiller (Unistat 815, Huber)
1000 W DC power supply
(XKW150-20, Xantrex)

PID Controller (2216e, Eurotherm)
GHP: 1000 W DC power supply

(GEN100-15, TDK Lambda)

Guards: 4x 480 W DC power supply (HSE480Ureg, Camtec)

2x PID Controller

(2604, Eurotherm)

750 W DC power supply (GEN30-25, TDK Lambda) / 2.4 kW DC electronic load (EA-EL 9400-50, Elektro-Automatic) 


\begin{tabular}{|c|c|}
\hline Measurement & Digital Multimeter (2700, Keithley) / Multiplexer (7708, Keithley) \\
\hline Force Application & $26 \mathrm{kN}$ linear actuator (Mini 3, Framo Morat) \\
\hline Force Measurement & Strain gauge force transducer (C9B, HBM) / Amplifier (AE301, HBM) \\
\hline Vacuum System & $\begin{array}{c}\text { Rotary vane pump (Trivac D65B, Leybold) / } \\
\text { Pumps }\end{array}$ \\
\hline Control & Curbomolecular pump (Turbovac TMP1000C, Leybold) \\
\hline Thermal \\
Measurement
\end{tabular}

\section{Investigation of heat flow measurements}

To the best of our knowledge, no comparison between experimental methods for heat flow determination on TEMs can be found in literature, which would include a standard-compliant description of involved measurement uncertainties for the variety of discussed metrological approaches. In this section we describe the uncertainty of heat flow measurements by means of the reference and absolute method. The uncertainty was investigated by measurements on a certified reference material for the thermal conductivity from the National Physical Laboratory (Inconel 600, NPL 2109) [27]. 


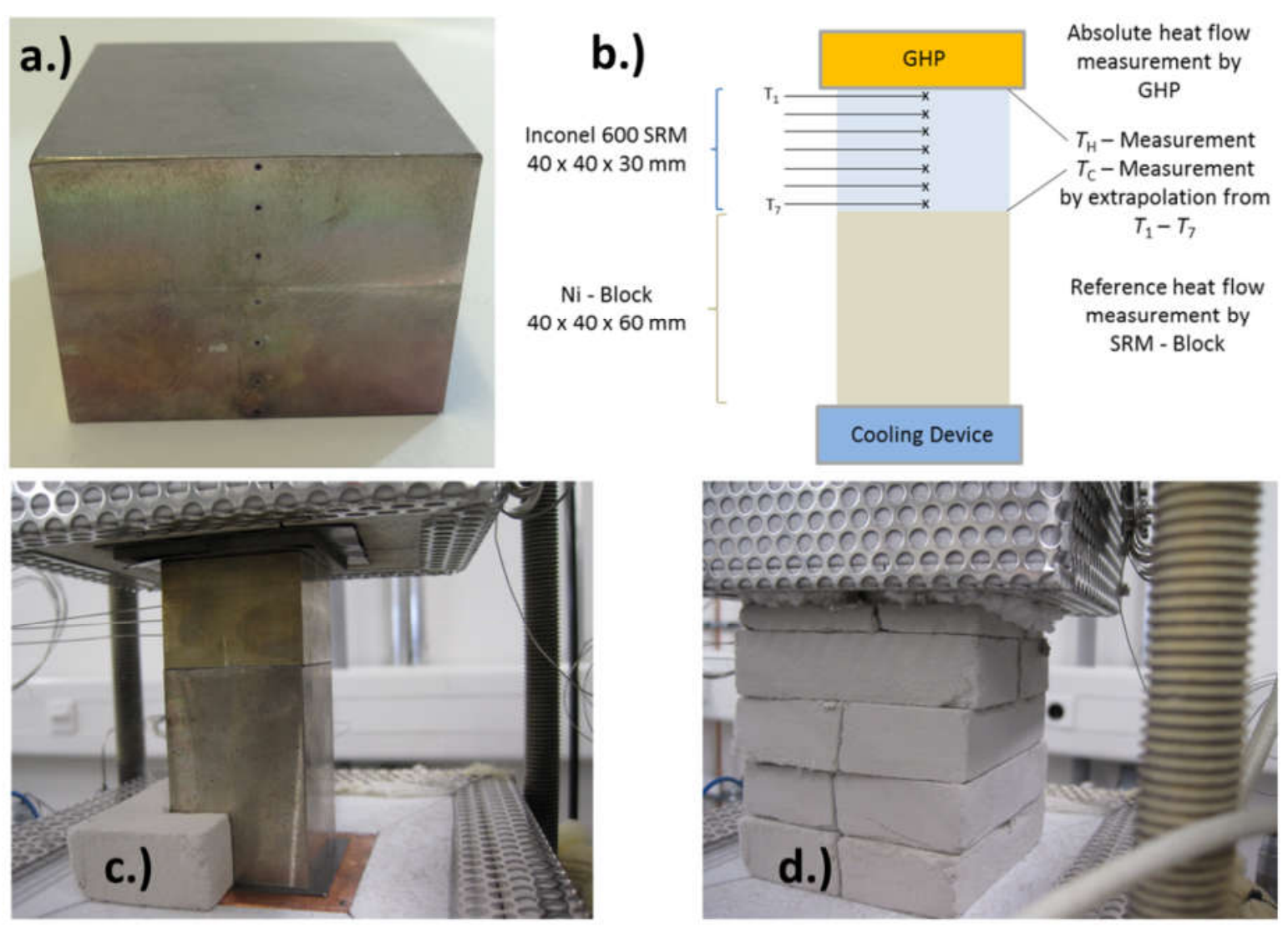

Figure 8: A HFM was manufactured from a standard reference material for the thermal conductivity (a) and tested within the A-TEGMA facility as shown in the schematic (b). The lower pictures show a corresponding installation prior (c) and after thermal insulation of the measuring section $(d)$.

The standard reference material (SRM) was certified in a temperature range between $373 \mathrm{~K}$ and $773 \mathrm{~K}$ with a relative uncertainty for the thermal conductivity $u\left(\kappa_{\mathrm{SRM}}\right)= \pm 4.8 \%(k=2)$. A HFM was manufactured from this material with a geometry of $40 \times 40 \times 30 \mathrm{~mm}^{3}$ and milled off and grinded to a tolerance of $\pm 0.1 \mathrm{~mm}$ in every dimension. Seven holes with a diameter of $0.5 \mathrm{~mm}\left( \pm_{0}^{0.05}\right)$ were eroded to a depth of $20 \mathrm{~mm}$ into the SRM-block to allow for temperature measurements along its vertical centreline. The five inner holes had a spacing of $5 \mathrm{~mm}$ to each other, while the two outer holes had a distance of $1 \mathrm{~mm}$ to the coupling areas. The temperature measurement was accomplished by seven type- $\mathrm{N}$ thermocouples (same type as mentioned above), which were inserted into the SRM-block with the use of heat-conducting paste (WLP300/200, Electronic Sensor) in order to improve their thermal coupling. $\Delta T_{\mathrm{M}}$ was determined from a linear extrapolation of temperatures obtained from these seven measuring points. This allowed for the determination of $T_{H}$ and $T_{C}$ within the SRM-block without the impact of thermal contact resistances on both coupling areas. The SRMblock was placed below the GHP-system of the A-TEGMA together with another Ni-Block $(40 \times 40 \times 60 \mathrm{~mm})$, in order to reproduce the usual installation conditions during TEM measurements (Figure 8). A $200 \mu \mathrm{m}$ thick graphite foil (Dr. Fritsch Sondermaschinen $\mathrm{GmbH}$ ) was used for thermal coupling between every component of the measuring section. In total, four temperature cycles (M01-M04) without intermediate re-installation of the SRM sample were conducted under vacuum $\left(<10^{-7} \mathrm{Bar}\right)$ at an axial pressure of $2 \mathrm{MPa}$. The temperature of the cooling plate was kept constant at $323 \mathrm{~K}$, while the GHP temperature $T_{\mathrm{GHP}}$ was varied between $373 \mathrm{~K}$ and $1023 \mathrm{~K}$ in every cycle. The minimum and maximum temperatures, which were reached during these experiments within the SRM-sample, exceeded the temperature range of certified values for the thermal conductivity by NPL. Thus, for heat flow evaluation in this work the thermal conductivity was derived from an 
extrapolation of the certified data by a parabolic fit. However, the approximated values were in excellent agreement to the certified ones in a wide temperature range of the conducted experiment and in a good agreement to a work of Blumm at al. [28], who has tested this reference material between $148 \mathrm{~K}$ and $1273 \mathrm{~K}$ with a combination of Laser Flash measurements, differential scanning calorimetry, and a dilatometer characterization. A comparison between the certified values, the reference data from [28] and the used approximation of thermal conductivity for heat flow determination in this study is shown in S1 (the prefix "S" denotes the supplementary information of this article).

In extension to the heat flow determination within the SRM-block by means of the reference principle in accordance to equation (5) and the absolute heat flow measurement by the GHP with the use of equation (6) an ANSYS $^{\circledR}$ finite element model (FEM) was used additionally to determine a reference value for the heat flow. The SRM-block was simulated as an isotropic body with same dimensions as used in the experiment and with first order boundary conditions on the base of the temperatures $T_{\mathrm{H}}$ and $T_{\mathrm{C}}$, which were experimentally obtained from a linear extrapolation of the seven measuring points within the SRM-block. The following table summarizes the simulated temperature conditions.

Table 2: Experimentally determined hot and cold side temperatures of the SRM-Block in dependence of the heater temperature. The hot and cold side temperatures have been applied as boundary conditions for the thermal FEM simulation of the heat flow through the SRM-Block.

\begin{tabular}{|c|c|c|}
\hline $\begin{array}{c}\text { Heater } \\
\text { Temperature [K] }\end{array}$ & $\begin{array}{c}\text { Hot Side Temperature of SRM- } \\
\text { Block [K] }\end{array}$ & $\begin{array}{c}\text { Cold Side Temperature of SRM- } \\
\text { Block [K] }\end{array}$ \\
\hline 373 & 360.08 & 328.51 \\
\hline 423 & 400.94 & 333.67 \\
\hline 473 & 444.68 & 343.35 \\
\hline 523 & 488.59 & 357.21 \\
\hline 573 & 531.9 & 372.26 \\
\hline 623 & 574.84 & 388.75 \\
\hline 673 & 616.99 & 406.55 \\
\hline 723 & 659.06 & 425.83 \\
\hline 773 & 700.72 & 446.04 \\
\hline 823 & 741.91 & 470.74 \\
\hline 873 & 783 & 491.84 \\
\hline 923 & 823.57 & 512 \\
\hline
\end{tabular}


The steady state heat flow simulation applied the approximated temperature dependency of the thermal conductivity of the SRM and did not consider side heat losses along the SRM-block (adiabatic conditions at lateral surfaces). Hexahedral Multiphysics elements (ANSYS: solid 226) with a maximum element length of $1 \mathrm{~mm}$ have been chosen for the mesh of the model. In total a minimum number of 48.000 elements have been used, which is in accordance to experience values from former mesh sensitivity analyses. The aim of the FEM simulation is to provide a reference value for the heat flow, which is based on experimental measurements of the hot and cold side temperature of the SRMblock only. These temperatures can be measured with relatively low uncertainty, which is close to the thermocouple specification and which equals approximately $1 \%$. By taking these temperatures into account, the simulation delivers a reference value of the heat flow without further disturbing influences from other impact variables. Section 3.3 contains the discussion of the experimental outcome and the comparison to results from FEM simulations. The uncertainty budgets for the reference and absolute heat flow determination are derived first in the following two sections.

\subsection{Uncertainty of the heat flow measurement by means of the reference principle}

The uncertainty of the heat flow measurement was determined in accordance to the "Guide to the expression of uncertainty in measurement" (GUM) [29]. The measurement function for the reference principle is based on a section-wise evaluation of the one-dimensional temperature gradient within the SRM-block.

$$
\dot{Q}_{R E F}=A_{S R M} \cdot \kappa_{S R M} \cdot\left|\nabla T_{S R M}\right|
$$

The combined uncertainty of the heat flow measurement $u\left(Q_{R E F}\right)$ is expressed by a geometric sum of terms, which represent individual uncertainty contributions of every input variable $X$ of the measurement function (equation 12). Every term consists of a product of the respective standard uncertainty $u(X)(k=1)$ and the corresponding sensitivity coefficient $c_{X}$. Each sensitivity coefficient equals the partial derivative of first order of the measurement function with respect to the respective input variable evaluated with best estimates of every input variable.

$$
\begin{gathered}
u\left(\dot{Q}_{R E F}\right)=\sqrt{c_{A}^{2} u\left(A_{S R M}\right)^{2}+c_{\kappa}^{2} u\left(\kappa_{S R M}\right)^{2}+c_{\nabla T}^{2} u\left(\left|\nabla T_{S R M}\right|\right)^{2}} \\
c_{A}=\frac{\partial \dot{Q}_{R E F}}{\partial A}=\kappa_{S R M} \cdot\left|\nabla T_{S R M}\right| \\
c_{\kappa}=\frac{\partial \dot{Q}_{R E F}}{\partial \kappa}=A_{S R M} \cdot\left|\nabla T_{S R M}\right| \\
c_{\nabla T}=\frac{\partial \dot{Q}_{R E F}}{\partial \nabla T}=A_{S R M} \cdot \kappa_{S R M}
\end{gathered}
$$

The standard uncertainties and best estimates of the input variables are either determined from experimental results or specifications from calibration certificates. In accordance to the given information within the SRM-certificate the standard uncertainty of the thermal conductivity of the SRM-block is $u\left(\kappa_{\text {SRM }}\right)= \pm 2.4 \%(k=1)$ at every temperature within the certification range. The best estimate is derived from the certified thermal conductivity at a respective temperature, which was determined from the average temperature within the interval between two temperature sensing 
points in the SRM-block. The standard uncertainty $u\left(A_{\text {SRM }}\right)$ relates to the cross section area and the manufacturing tolerance of the block and is expressed in a sub-model, which follows the same procedure. The measurement function for $A_{R E F}=W \cdot L$ is given by the multiplication of the width $(W)$ by the length $(L)$ of the SRM-block (dimensions perpendicular to the direction of heat flow). The uncertainty contributions for these lateral dimensions combine best estimates for $W$ and $L$ (each $40 \mathrm{~mm}$ ) with their standard uncertainties $u(W)$ and $u(L)$, which correspond to the manufacturing tolerance of $\pm 0.1 \mathrm{~mm}$.

$$
\begin{gathered}
u\left(A_{S R M}\right)=\sqrt{c_{W}^{2} u(W)^{2}+c_{L}^{2} u(L)^{2}} \\
c_{W}=\frac{\partial A_{R E F}}{\partial W}=L \\
c_{Y}=\frac{\partial A_{R E F}}{\partial L}=W
\end{gathered}
$$

The best estimates and standard uncertainties for the temperature gradient were taken from an evaluation of the respective temperature profiles, which were measured within the SRM-block and linearly fitted in accordance to the measurement function, which implies a linear temperature profile due to a one-dimensional (1D) heat flow. Exemplary results are given for the outcome of the first temperature cycle (M01) at two different temperatures of the GHP ( $\left.T_{G H P}\right)$ within Figure 9 . According to this evaluation the standard uncertainty at $T_{\mathrm{GHP}}=100^{\circ} \mathrm{C}$ was $u(\nabla T)=44.88 \mathrm{~K} / \mathrm{m}$, which corresponds to $\pm 3.88 \%$ of the best estimate $\nabla T=1155.43 \mathrm{~K} / \mathrm{m}$. The standard uncertainty at $T_{\mathrm{GHP}}=750^{\circ} \mathrm{C}$ was $u(\nabla T)=226.89 \mathrm{~K} / \mathrm{m}$, which is $\pm 1.77 \%$ of the best estimate $\nabla T=12789.61 \mathrm{~K} / \mathrm{m}$.
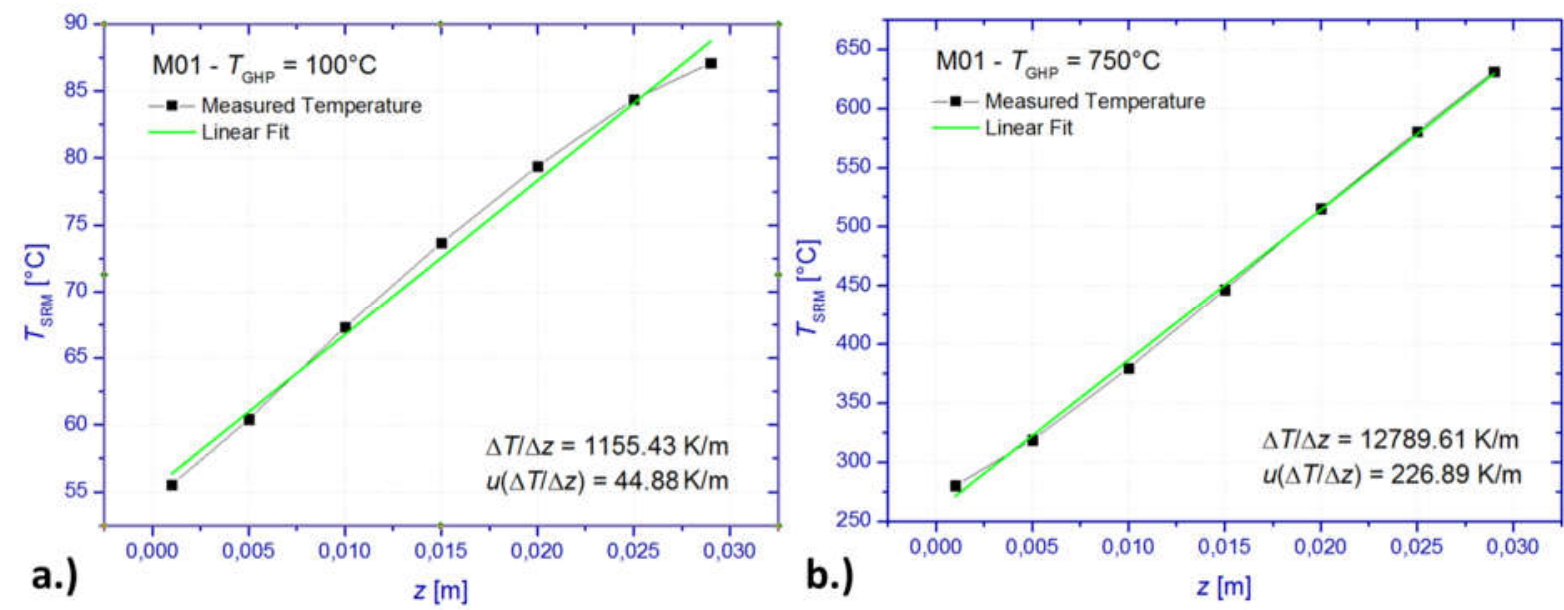

Figure 9: Measured temperature profiles within the SRM-block (full black symbols) with corresponding linear fits (green line) and disclosure of the best estimates and standard uncertainties of the temperature gradient at a GHP temperature of $T_{\mathrm{GHP}}=100^{\circ} \mathrm{C}(\mathrm{a})$ and $T_{\mathrm{GHP}}=750^{\circ} \mathrm{C}(\mathrm{b})$.

The evaluation of the combined uncertainty for the heat flow determination by means of the reference principle was accomplished in accordance to equation (13) for every single of the four conducted measurements. Results for the individual test runs can be found in S2. The combined uncertainty $u\left(Q_{\mathrm{REF}}\right)$ increases with temperature (Figure 10) since the uncertainty contributions for the temperature gradient and the thermal conductivity of the SRM increase, too. This is a result of the monotonous increase of corresponding standard uncertainties and best estimates for these input variables. The relative uncertainty is highest at lowest temperature gradients and heat flow. Overall, the mean value of the relative uncertainty is in the range between $10 \%<u\left(Q_{R E F}\right)<13 \%$. 


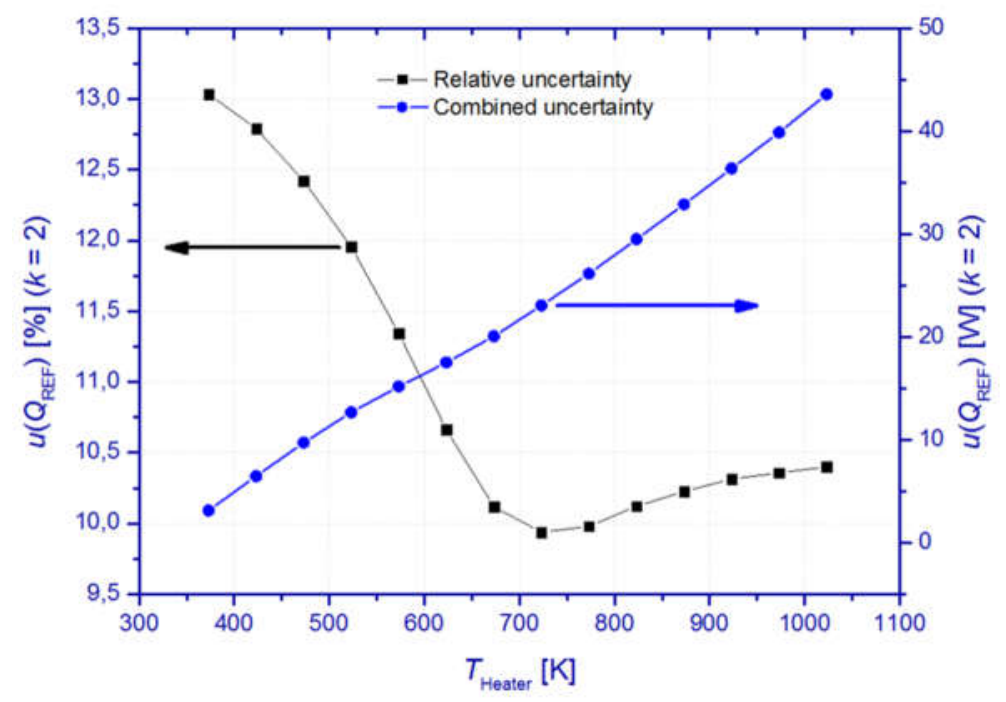

Figure 10: Relative uncertainty (black squares) and combined uncertainty (blue circles) of the heat flow measurement by means of the reference principle. The shown results correspond to the mean values from four measurements on a HFM, which was manufactured from the Inconel 600 reference material for thermal conductivity of the NPL.

\subsection{Uncertainty of the GHP-based absolute heat flow determination}

The uncertainty of a GHP-based measurement of heat flow could be principally derived from a similar procedure as described for the reference principle. This would involve uncertainty contributions for the measurements of the electric current and the voltage drop over the GHP in accordance to equation (6), which represents the underlying measurement function. In fact these electric measurands can be determined with very low uncertainty due to low standard uncertainties of appropriately chosen measurement hardware like low tolerance shunt resistors for current measurement and high quality voltmeters. However, this approach does not give a representative view on the uncertainty of a GHP-based characterization, since its uncertainty is mainly determined by the thermal cross talk between the GHP and the guard heaters.

ASTM-C177 [23] describes the gap imbalance error, which is introduced by the temperature difference between the GHP and the guard heaters, as one origin of a heat exchange between the GHP and the guards. The systematic contribution to this error is given by the fact, that only a finite number of sensor locations can be used for temperature imbalance detection. Thus, even if the temperature differences between opposing sides of the gaps are reduced to zero will not necessarily ensure a vanishing net heat flow across these gaps. Consequently the systematic error component is influenced by the sensor positioning and the signal to noise ratio within the sensor circuits, while short-term fluctuations of the temperature control introduce an additional random component to the gap imbalance error.

ASTM-C177 suggests two ways to determine the impact of the gap imbalance error on the uncertainty for heat flow measurements. First, the exchanged heat flow between the GHP and the guard heaters can be calculated. In fact this procedure is already very challenging for the characterization of homogeneous material samples only, but becomes extremely demanding for the case of a GHP-based measurement on TEMs due to an increasing number of influencing parameters and difficulties in determination of elusive thermal coupling conditions between the components of the GHP-system and along externally attached parts of the measuring section. While design-inherent parameters (gap geometry, insulating gap fill material, emittance of gap surfaces, mechanical 
support, and number/type of crossing wires) have to be considered anyway, the significant difference to a TEM characterization is given by the fact that the axial pressure, means for TEM coupling, the heat flow through the TEM, and its geometry must be variable over a wide range for a versatilely applicable characterization device. Consequently the calculation of the gap imbalance error seems to represent at least a laborious way to specify the associated measurement uncertainty and poses inaccurate predictions in case of lacking knowledge on tolerances and interdependencies of influencing parameters.

According to ASTM-C177 the second way is given by an empiric determination of the gap imbalance error. This procedure suggests a measurement of the dependence of the GHP power change $\delta Q_{G H P}$

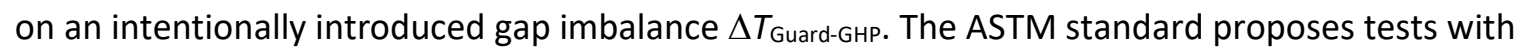
three different temperature settings, one with a balanced guard and one each biased positive and negative. The introduced temperature imbalance should be high enough to allow for a conclusive measurement of $\delta Q_{\mathrm{GHP}}$ but still low enough to remain a linear dependence of $\delta Q_{\mathrm{GHP}}$ upon the

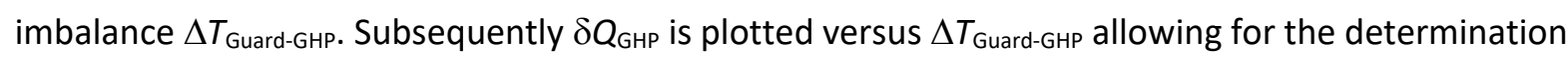
of the effective conductance $X_{\text {Guard-GHP }}$ (Figure 11 ) between the GHP and its guards from the slope of the linear approximation of $\delta Q_{G H P} / \Delta T_{\text {Guard-GHP. }}$

$$
X_{\text {Guard-GHP }}=\frac{\delta Q_{G H P}}{\Delta T_{\text {Guard }-G H P}}=\frac{\delta Q_{G H P}}{T_{\text {Guard }}-T_{G H P}}
$$

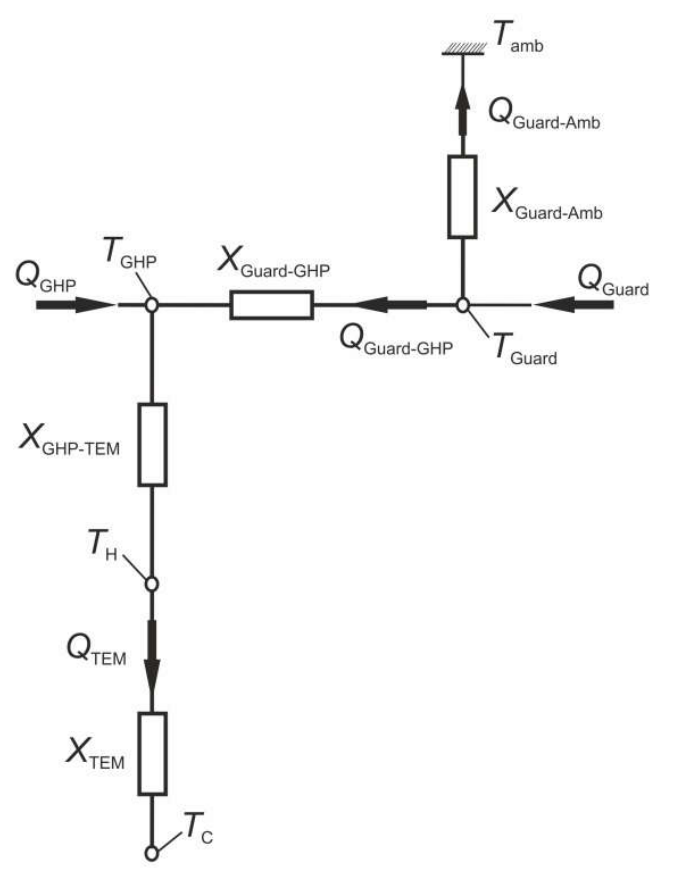

Figure 11: The thermal equivalent circuit of the GHP-apparatus involves temperatures and the generated heat flow of the GHP and the guard heaters. The conductances represent the thermal coupling between the components of the GHPsystem. $T_{\text {amb }}$ represents the temperature of the environment of the measuring section. The guard heaters are coupled to the environment through their outer insulation, which is represented by $X_{\text {Guard-Amb. }}$.

In this study $T_{\mathrm{GHP}}$ was varied between $323 \mathrm{~K}$ and $773 \mathrm{~K}$ in steps of $50 \mathrm{~K}$. At every $T_{\mathrm{GHP}}$ the temperatures of the guard heaters $T_{\text {Guard }}$ were detuned by $\Delta T_{\text {Guard-GHP }}= \pm 10 \%$ of $T_{\mathrm{GHP}}$ in at least five steps. The relative value of $\Delta T_{\text {Guard-GHP }}$ relates to $T_{\mathrm{GHP}}$ in [ ${ }^{\circ} \mathrm{C}$ ]. After every change of $T_{\mathrm{GHP}}$ or $T_{\text {Guard }}$ a stabilization time of $3 \mathrm{~h}$ was used to ensure thermal steady state conditions for precise heat flow measurements. This led to a total duration of $183 \mathrm{~h}$ for this experiment. 

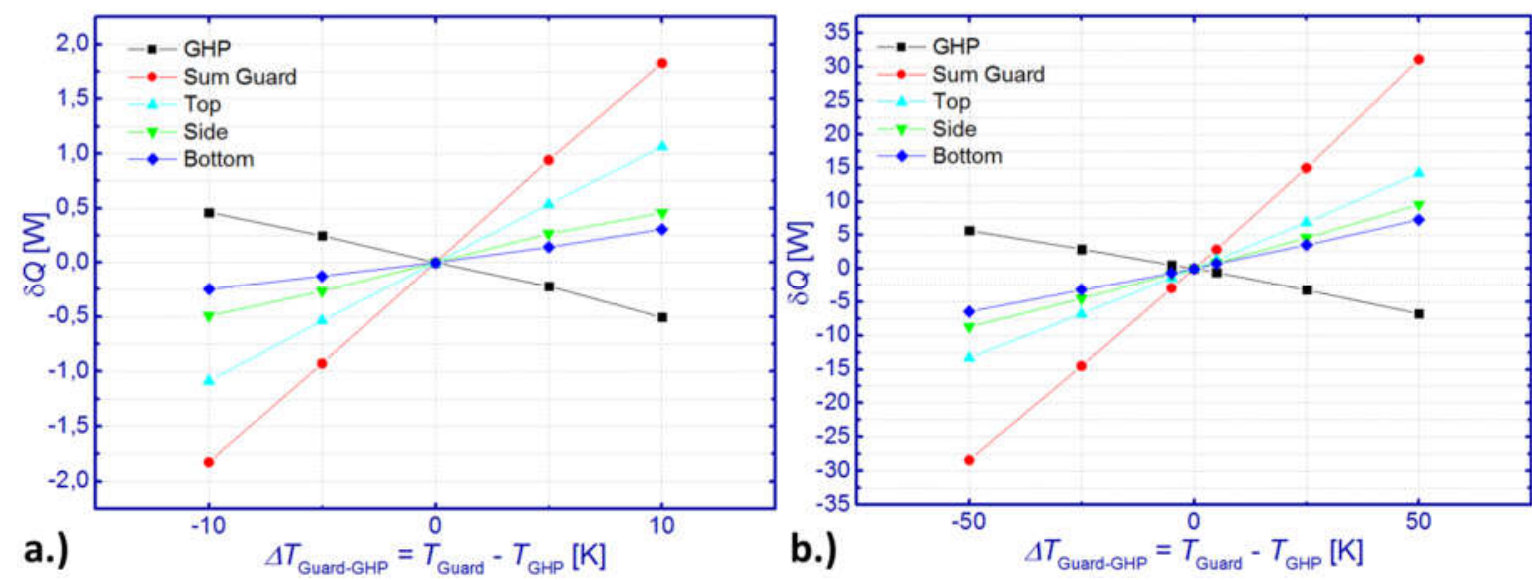

Figure 12: Experimentally determined sensitivity of the metered GHP power (black squares) on an intentionally provoked thermal imbalance between $T_{\mathrm{GHP}}$ and $T_{\mathrm{Guard}}$ at $T_{\mathrm{GHP}}=373 \mathrm{~K}(\mathrm{a})$ and $T_{\mathrm{GHP}}=773 \mathrm{~K}(\mathrm{~b})$. Additionally to the change of the GHP power the change of the guard heater power is displayed individually for the top (cyan triangles), side (green triangles) and bottom heater (blue rhombs) and as the sum of all guard heater circuits (red circles).

Exemplary results of this experiment at $T_{\mathrm{GHP}}=373 \mathrm{~K}$ and $T_{\mathrm{GHP}}=773 \mathrm{~K}$ (Figure 12) show that for a positive imbalance ( $T_{\text {Guard }}>T_{\mathrm{GHP}}$ ) $\delta Q_{\mathrm{GHP}}$ becomes negative in relation to the balanced point at $T_{\mathrm{GHP}}=T_{\text {Guard }}$ and changes its sign when the sign of imbalance changes. The top guard heater shows systematically the highest power consumption among all guards, as it is the only one with a good thermal coupling to the environment of the measuring section due to its direct attachment to the mechanical support of the GHP-system. The experimental quality in terms of temperature control and power measurement on the individual heater circuits can be assessed by the linear dependence

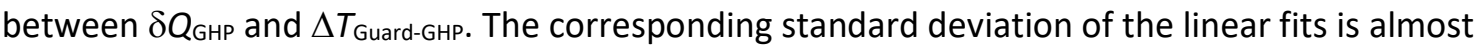
constant $\sigma\left(\delta Q_{\mathrm{GHP}} / \Delta T_{\text {Guard-GHP}}\right)=2.5 \%$ for the tested range of $T_{\mathrm{GHP}}$. Using equation (20) gives access to the effective thermal conductance $X_{\text {Guard-GHP }}$ between the GHP and the guard heater system (Figure 13).

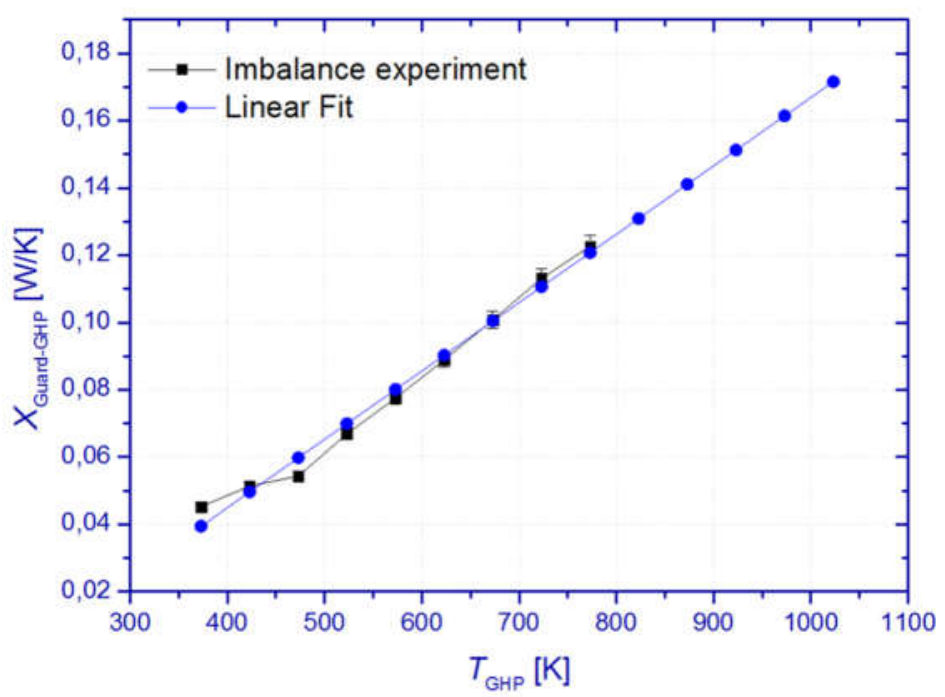

Figure 13: Effective thermal conductance $X_{\text {Guard-GHP }}$ between the GHP and the guard heater system, obtained from an imbalance experiment for a GHP temperature range between $373 \mathrm{~K}<T_{\mathrm{GHP}}<773 \mathrm{~K}$ (black squares). The figure shows a linear approximation of $X_{\text {Guard-GHP }}$ up to $1023 \mathrm{~K}$ (blue circles). 
The effective heat flow through the TEM $Q_{\text {TEM }}$ corresponds to the sum of the heat flow, which is generated by the GHP $Q_{G H P}$, and positive or negative contributions from the guard heater system Q Guard-GHP.

$$
Q_{T E M}=Q_{G H P}+Q_{\text {Guard }-G H P}=Q_{G H P}+X_{\text {Guard }-G H P} \cdot\left(T_{\text {Guard }}-T_{G H P}\right)
$$

The contribution from the guard heater system to $Q_{\text {TEM }}$ represents the uncertainty of the GHP-based heat flow determination, which is determined by $X_{\text {Guard-GHP }}$ and the effective temperature difference between $T_{\mathrm{GHP}}$ and $T_{\text {Guard }}$ under nominally balanced temperature conditions. A significant difference between ASTM-C177 and a GHP-based characterization of the heat flow through a TEM comes to the fore at this point, which impairs a direct applicability of this standard. ASTM-C177 relates to measurements on homogeneous material samples and even more decisive under relatively low temperature differences at the tested specimens of a few tens of $K$ only. By use of thermally high conductive materials for the construction of the GHP, ASTM-C177 assumes an almost constant $T_{\text {GHP }}$ within the guarded zone. A remaining non-uniformity of $T_{\mathrm{GH}}$ is defined as the origin of the so called edge heat loss error. According to ASTM-C177, results of reported studies $[30,31,32,33]$ are not proven to a level, which would allow for an universally accepted correction of this error. However, significantly higher heat flow and temperature differences are needed for a meaningful characterization of TEMs compared to a usual GHP-based characterization on a material sample. Thus, a considerable temperature difference $\Delta T_{\text {GHP-TEM }}=T_{\text {GHP }}-T_{H}$ can be introduced between the GHP and the hot side of the TEM by $Q_{\text {TEM, }}$ which leads to an inevitable thermal imbalance between the heat flow path from the GHP to the TEM and the guard heater system. $\Delta T_{\text {GHP-TEM }}$ drops at the Cushell, which is located between the GHP and the TEM sample and represented by $X_{\text {GHP-TEM }}$ within the thermal equivalent circuit.

$Q_{\text {TEM }}$ and the temperature drop $\Delta T_{\text {GHP-TEM }}$ at $X_{\text {GHP-TEM }}$ determine the effective thermal imbalance between the GHP and the guard heaters. In order to quantify the overall uncertainty of the GHPbased heat flow measurement $\Delta T_{\text {GHP-TEM }}$ was determined experimentally for $373 \mathrm{~K}<T_{\text {GHP }}<1024 \mathrm{~K}$ in the course of four repetitive measurements with nominally balanced temperature conditions between the GHP and the guard heaters. According to this experiment $\Delta T_{\text {GHP-TEM }}$ equals between $1 \mathrm{~K}$ and $18 \mathrm{~K}$ (see S.3) in dependence of $T_{\mathrm{GHP}}$ and $Q_{\mathrm{TEM}}$, respectively. The uncertainty of the heat flow measurement $u\left(Q_{\mathrm{GHP}}\right)$ was determined by setting $\Delta T_{\text {GHP-TEM }}=\Delta T_{\text {Guard-GHP }}$ in equation (21).

$$
u\left(\dot{Q}_{G H P}\right)=\dot{Q}_{\text {Guard-GHP}}=X_{\text {Guard }-G H P} \cdot\left(\Delta T_{G H P-T E M}\right)
$$


The combined uncertainty $u\left(Q_{G H P}\right)$ increases with temperature (Figure 14$)$ since $\Delta T_{\text {GHP-TEM }}$ and $X_{\text {GHP-TEM }}$ increase, too. This is a result of the monotonous increase of corresponding standard uncertainties and best estimates for these input variables. Overall, the mean value of the relative uncertainty is in the range between $0.2 \%<u\left(Q_{\mathrm{GHP}}\right)<0.75 \%(k=2)$. At this point is has to be stated that the uncertainty of the absolute method is based on findings from the thermal imbalance experiment, which was conducted up to $773 \mathrm{~K}$ only due to the increased time required. Consequently the uncertainty of the absolute method is experimentally founded up to $773 \mathrm{~K}$, whereas only a linear extrapolation of $X_{\text {GHP-TEM }}$ (Figure 13) builds the base for the temperature interval between $773 \mathrm{~K}$ and $1023 \mathrm{~K}$.

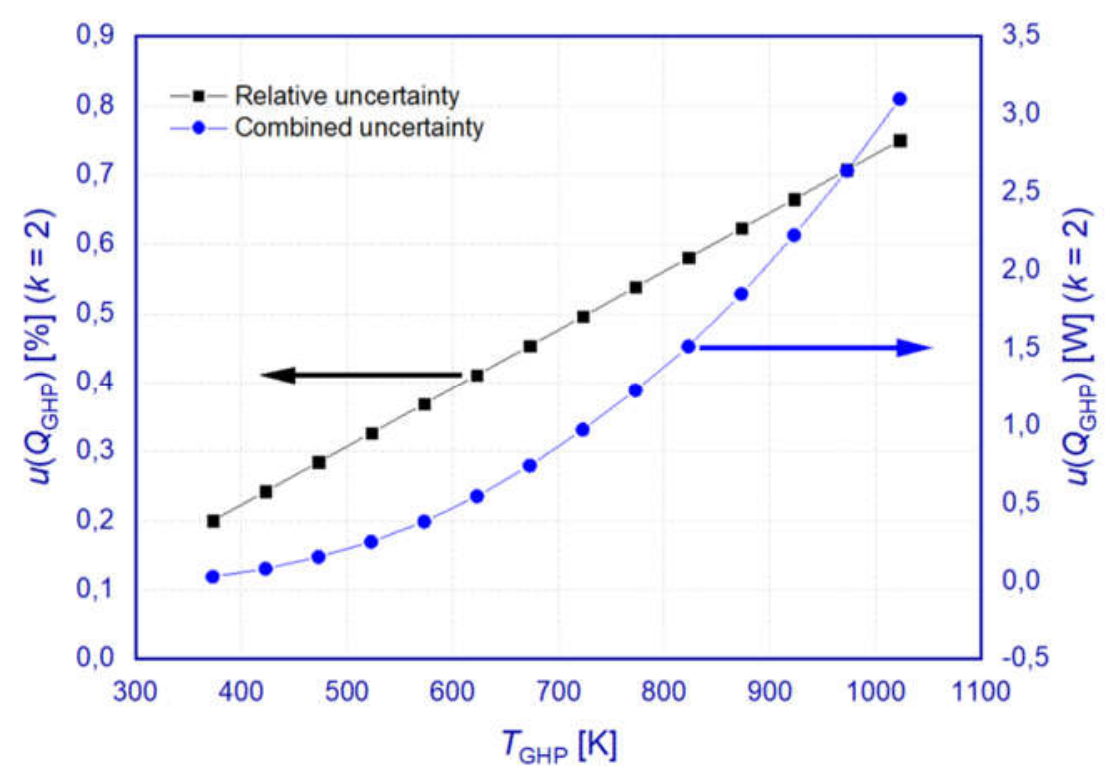

Figure 14: Relative uncertainty (black squares) and combined uncertainty (blue circles) of the heat flow measurement by means of the absolute principle.

\subsection{RESULTS AND DISCUSSION}

This section discusses heat flow results, which were obtained on the SRM-Block with the described configuration of the measuring section (Figure 8 ) in the course of four temperature cycles (M01 M04) between $373 \mathrm{~K}<T_{\mathrm{GHP}}<1023 \mathrm{~K}$ at $T_{\mathrm{C}}=323 \mathrm{~K}$. The discussion involves $Q_{\mathrm{REF}}$ as the result of the applied reference principle within the SRM-Block, $Q_{\mathrm{GH}}$ as the outcome of the absolute method, and a

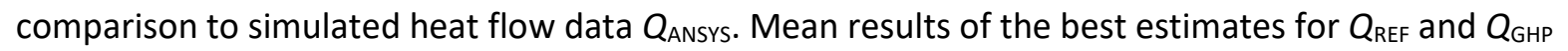
from every temperature cycle revealed a decent accordance with $Q_{\text {ANSYS }}$ (Figure 15a) showing a relatively low deviation and a good reproduction of the temperature characteristic of the heat flow. Results of individual temperature cycles can be found in S4. The displayed error bars in Figure 15a correspond to the relative uncertainties of the reference principle (Figure 10) and the absolute method (Figure 14). Since $Q_{\text {Ansys }}$ was determined from FEM simulation of the SRM-block without tolerances for $A_{S R M}$, the uncertainty $u\left(Q_{A N S Y S}\right)$ is based only on the uncertainty contribution of the thermal conductivity of the SRM-Block. An additional uncertainty of the extrapolated temperatures of the coupling areas of the SRM-Block, which were taken as boundary temperatures for the FEM simulation of heat flow, was not considered. 

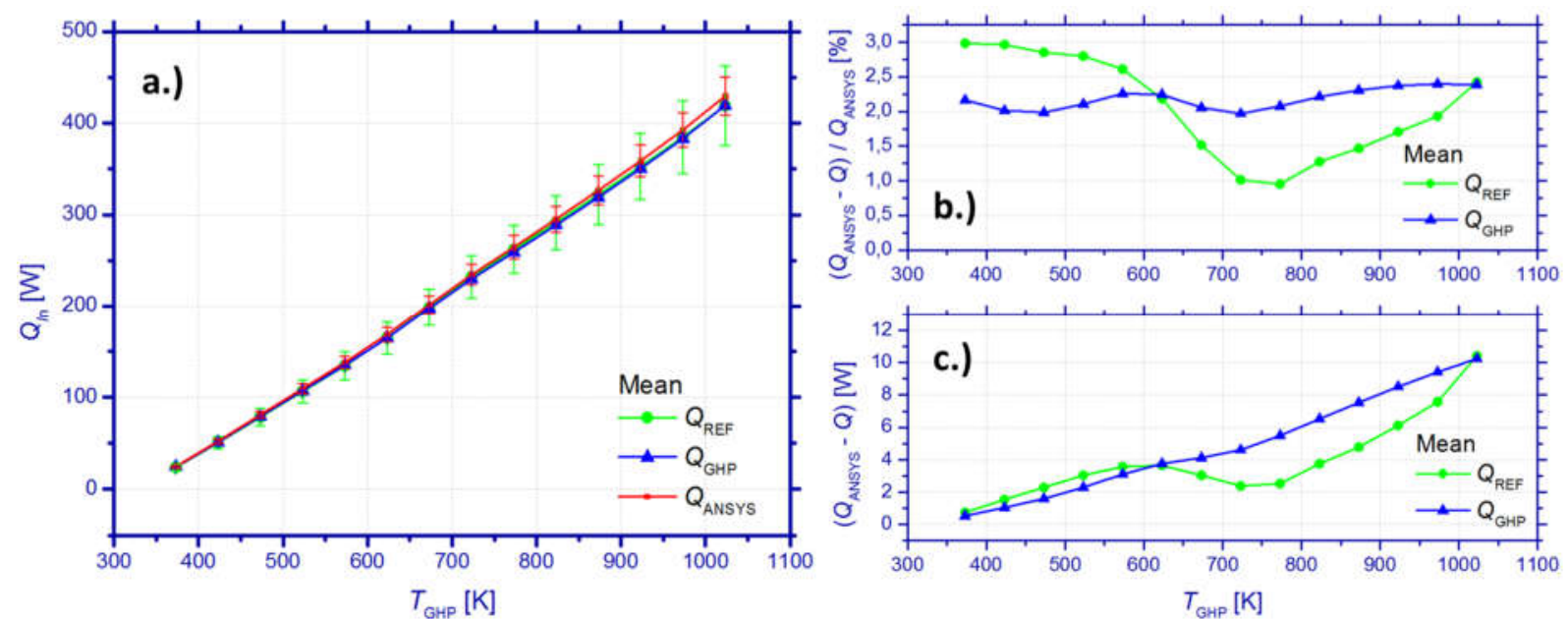

Figure 15: Mean heat flow results obtained by the reference principle $\left(Q_{\mathrm{REF}}\right)$ and an absolute method $\left(Q_{\mathrm{GHP}}\right)$ in comparison to simulated data $\left(Q_{\mathrm{ANSYS}}\right)$ of four repetitive measurements on an Inconel 600 reference material for the thermal conductivity (a). Additionally, the relative (b) and absolute (c) deviations between $Q_{\text {ANSYS }}$ and $Q_{\text {REF }}$ and $Q_{\mathrm{GHP}}$, respectively, are shown.

The relative deviation between $Q_{\text {ANSYS }}$ and the mean from the best estimates for $Q_{\text {REF }}$ and $Q_{\text {GHP, }}$ respectively, is below $3 \%$ for the entire temperature interval (Figure $15 \mathrm{~b}$ ). $Q_{\mathrm{GHP}}$ reveals an almost temperature independent relative deviation to $Q_{\mathrm{ANSYS}}$, whereas the relative difference of $Q_{\mathrm{REF}}$ shows an irregular course of deviation, which is qualitatively reproducing the temperature dependency of the uncertainty of heat flow determination of the reference principle (Figure 10), too. Up to medium temperatures $\left(T_{\mathrm{GHP}}<780 \mathrm{~K}\right)$ the observed variation of $\Delta Q_{\mathrm{ANSYS}-\mathrm{REF}}=\left(Q_{\mathrm{ANSYS}}-Q_{\mathrm{REF}}\right)$ might be a result of a fluctuating thermal coupling between the thermocouples and the SRM-Block. At lower temperatures the coupling is governed by heat conduction. A weak and undefined sensor contact can yield a locally lowered temperature measurement and the possibility to increase the best estimate of the evaluated temperature gradient, which could hold for the higher $Q_{\mathrm{REF}}$ at $T_{\mathrm{GHP}}<600 \mathrm{~K}$. With increasing mean temperature of the SRM-Block the sensor coupling shows a successively stronger radiative component, which can compensate an irregular contact between particular sensors and the SRMBlock. This could yield a flattening of the measured temperature profile, which coincides with the decrease of $\Delta Q_{\text {ANSYS-REF }}$ in the middle temperature range $600 \mathrm{~K}<T_{\text {GHP }}<780 \mathrm{~K}$. The increase of $\Delta Q_{\text {ANSYS- }}$ REF for $T_{\mathrm{GHP}}>780 \mathrm{~K}$ is most likely a result of the successive heat loss along the SRM-Block by radiation. This assumption is supported by the observable bending of the temperature profile within the SRMBlock and its steeper course at higher temperatures (Figure 9b). Due to the increased temperature gradient $Q_{\text {REF }}$ shows an increase towards higher temperatures again. A relative difference $<3 \%$ corresponds to an absolute deviation $<10.4 \mathrm{~W}$ (Figure 15c). The deviation $\Delta Q_{\text {ANSYS-REF }}$ for $T_{\text {GHP }}>615 \mathrm{~K}$ is even smaller compared to $\Delta Q_{\text {ANSYS-GHP }}=\left(Q_{\text {ANSYS }}-Q_{G H P}\right)$. However, these values reflect only the best estimates but not the underlying uncertainty, which is lowest for the GHP-based absolute method (Figure 16). 


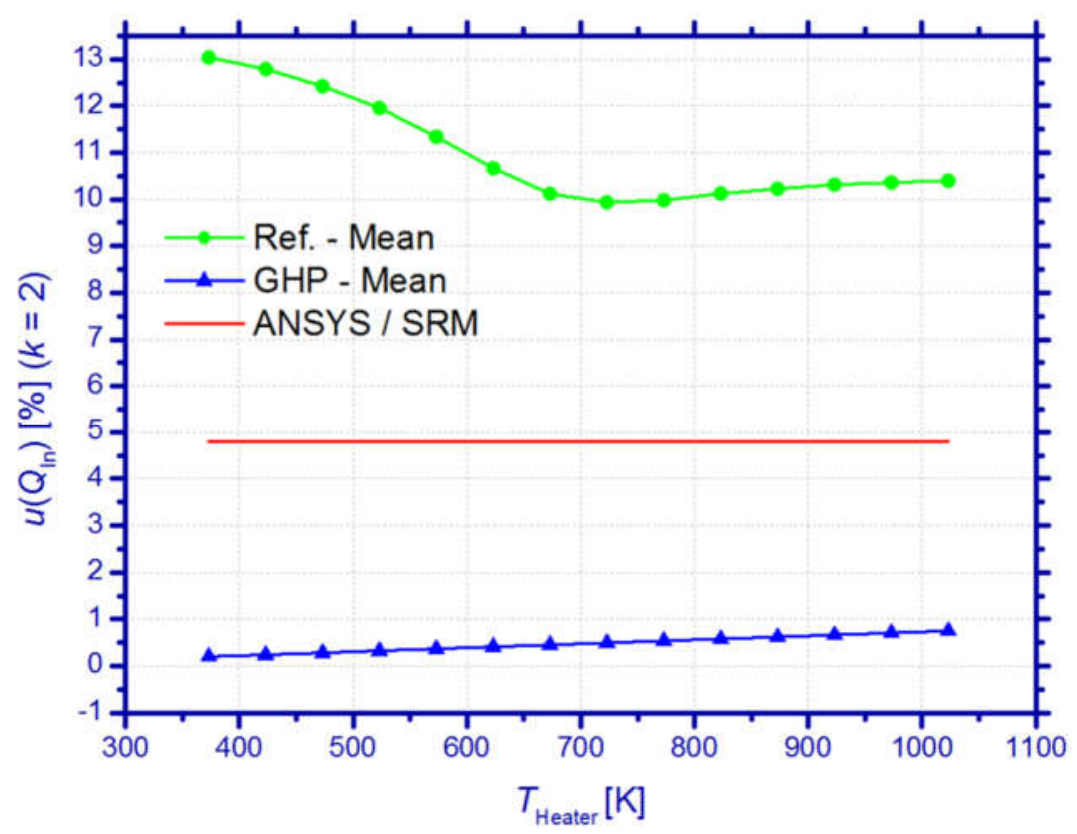

Figure 16: Uncertainty of the heat flow measurement of the reference principle (full green circles) and the GHP-based absolute method (full blue triangles) in comparison to the uncertainty of simulated heat flow, which only relates to the uncertainty contribution of the thermal conductivity of the testes standard reference material.

The present study confirms a significantly lower uncertainty of the absolute method compared to the reference principle. The best estimates for the heat flow show a decent accordance among both methods and in comparison to the reference value obtained from FEM simulations. However, the absolute method reveals an almost temperature independent deviation (Figure 15b) to the simulated heat flow, whereas a distinct temperature characteristic can be observed for the deviation of the best estimates of the reference principle, which coincides with the temperature dependency of the standard deviation of the thermal gradient within a HFM. Its temperature characteristic is in turn caused by a temperature dependent thermal sensor coupling within the HFM and parasitic effects such as inhomogeneous coupling conditions at interfaces of the measuring section or lateral heat losses, which lead to a deviation from one-dimensionality of the heat flow within a HFM.

Consequently it can be assumed that the resulting deviation of the reference principle is prone to show a significantly higher sensitivity to sample and sensor installation conditions than the absolute method.

Apart from the resulting deviation and uncertainty for heat flow determination, which is the focus of this work, users may prioritize other features of characterization devices like measurement speed, or costs and complexity of measurement instrumentation. These features may vary in dependence of the specific system implementation. However, due to the higher number of heating circuits, sensors and the necessity of longer stabilization times the absolute method appears to be more complex and laborious than the reference principle.

\section{CONCLUSIONS}

A generic procedure for the characterization of TEM was suggested. The procedure and the described analytic expressions for determination of particular measurands cover the full spectrum of functional TEM properties, which are needed for a benchmarking on TEMs and the development of thermoelectric generator systems for energy harvesting applications. Special focus was placed in this study on two methods for heat flow determination. Full uncertainty budgets were determined for 
the reference principle and a GHP-based absolute method on the base of measurements on a standard reference material for the thermal conductivity. Both methods deliver good accordance to the outcome of a comparative FEM simulation showing relative deviations for the heat flow $<3 \%$ within the investigated temperature range up to $1023 \mathrm{~K}$. The difference between the reference and the absolute method is given by their uncertainties. Decisive uncertainty contributions of the reference principle are given by the uncertainty of the thermal conductivity of the used HFM and the uncertainty of the measured temperature gradient. The evaluation showed a combined uncertainty between $10 \%<u\left(Q_{\mathrm{REF}}\right)<13 \%(k=2)$ for the reference principle. The uncertainty of the GHP-based absolute method is mainly determined by the thermal cross talk between the metering heater and the guard heaters. Experiments with provoked thermal imbalance allowed for a quantification of the effective thermal conductance between these heaters. The high demand on heat flow, which is needed for a meaningful TEM characterization, yields an inevitable thermal imbalance even under nominally balanced condition. The maximum occurring thermal imbalance was determined experimentally and combined with the effective thermal conductance ( $X_{\text {Guard GHP }}$ ) for the investigation of the resulting uncertainty. According to these results the absolute method showed a combined uncertainty between $0.2 \%<u\left(Q_{\mathrm{GHP}}\right)<0.75 \%(k=2)$. In the temperature range between $773 \mathrm{~K}$ and $1023 \mathrm{~K}$ the uncertainty of the absolute method is based on a linear extrapolation of $X_{\text {Guard GHP }}$ only. In order to improve the confidence level future works will have to validate these findings experimentally as it was accomplished in this study for the temperature interval between $373 \mathrm{~K}$ and $773 \mathrm{~K}$. Further measurements have to be accomplished in order to increase the confidence level with regard to statistic distributions of particular measurands and influencing parameters, too. In this course various parameters (sample geometry, means for thermal coupling, axial pressure, sensor count and installation) have still to be investigated in order to obtain a comprehensive view on the uncertainty of particular characterization and evaluation methods. Special regard has to be paid on the coverage of a meaningful property range for investigated samples. A versatile metrology with applicability for various types of TEM should be characterized by a given traceability and specified uncertainty budgets for any relevant range of measurands. Apart from the uncertainty of heat flow determination the investigated methods are marked by differences of their complexity, costs and measurement speed, too. These features have not been addressed in this work, but will most likely reveal advantages for the reference principle, since the absolute method is characterized by a higher complexity and relatively longer stabilization times for thermal equilibrium. User with a focus on a fast and simple TEM characterization may choose the application of the reference principle but should be aware of the underlying uncertainty of their measurements.

\section{ACKNOWLEDGEMENTS}

Financial support is gratefully acknowledged for the project "Thermoelectric Standardization for High Temperatures" (TEST-HT, grant number 03VP04401), which was granted by the German Federal Ministry of Education and Research.

\section{REFERENCES}

[1] D. M. Rowe, "Chapter 1. General Principles and Basic Considerations " in Rowe, D. M.(ed.), Thermoelectric Handbook - Macro to Nano, Boca Raton, USA: Taylor \& Francis Group, 2006, p. 1-1. doi:10.1201/9781420038903.sec1

[2] Ioffe, A. F.; Stilbans, L. S.; lordanishvili, E. K.; Stavitskaya, T. S.; Gelbtuch, A.; Vineyard, G. Semiconductor Thermoelements and Thermoelectric Cooling. Physics Today 1959, 12:42. 
[3] Salvador, J.R.; Cho, J.Y.; Ye, Z.; Moczygemba, J.E.; Thompson, A.J.; Sharp, J.W.; König, J.D.; Maloney, R.; Thompson, T.; Sakamoto, J.; Wang, H.; Wereszczak, A.A.; Meisner, G.P. Thermal to Electrical Energy Conversion of Skutterudite-Based Thermoelectric Modules. J. of Elec Materi 2013, Vol. 42:1389, https://doi.org/10.1007/s11664-012-2261-9

[4] Ochi, T.; Nie, G.; Suzuki, S.; Kikuchi, M.; Ito, S.; Guo, J.Q. Power-Generation Performance and Durability of a Skutterudite Thermoelectric Generator. Journal of Elec Materi. 2014, Vol. 43:2344, doi:10.1007/s11664-014-3060-2

[5] Garcia-Canadas, J.; Powell, A.V.; Kaltzoglou, A.; Vaqueiro, P.; Min, G. Fabrication and Evaluation of a Skutterudite-Based Thermoelectric Module for High-Temperature Applications. Journal of Elec Materi 2013, Vol. 42:1369, https://doi.org/10.1007/s11664-0122241-0

[6] Bartolomé, K.; Balke, B.; Zuckermann, D.; Köhne, M.; Müller, M.; Tarantik, K.; König, J. Thermoelectric Modules Based on Half-Heusler Materials Produced in Large Quantities. Journal of Elec Materi 2014, Vol. 43:1775, https://doi.org/10.1007/s11664-013-2863-x

[7] TEC TEG MFR. - https://thermoelectric-generator.com/teg-cascade-800c-hot-sidethermoelectric-power-modules/ - Retrieved: 2019-09-17

[8] Novus Energy Technologies http://www.novusenergytechnologies.com/technologies/power_generation.html Retrieved: 2019-09-17

[9] Kryotherm - http://kryothermtec.com/medium-temperature-generating-modules-marsseries.html - Retrieved: 2019-09-17

[10] Joshi, G.; Poudel, B. Efficient and Robust Thermoelectric Power Generation Device Using Hot-Pressed Metal Contacts on Nanostructured Half-Heusler Alloys. Journal of Elec Materi 2016, Vol. 45:6047, doi:10.1007/s11664-016-4692-1

[11] Zhang, Q.; Liao, J.; Tang, Y.; Gu, M.; Ming, C.; Qiu, P.; Bai, S.; Uher, C.; Chen, L. Realizing a thermoelectric conversion efficiency of $12 \%$ in bismuth telluride/skutterudite segmented modules through full-parameter optimization and energy-loss minimized integration. Energy Environ. Sci. 2017, Vol. 10:956, doi:10.1039/C7EE00447H

[12] Chetty, R.; Nagase, K.; Aihara, M.; Jood, P.; Takazawa, H.; Ohta, M.; Yamamoto, A. Mechanically durable thermoelectric power generation module made of $\mathrm{Ni}$-based alloy as a reference for reliable testing. Applied Energy 2020, 114443, https://doi.org/10.1016/j.apenergy.2019.114443.

[13] Marchi A.D.; Giaretto, V. An accurate new method to measure the dimensionless figure of merit of thermoelectric devices based on the complex impedance porcupine diagram. Rev. of Scientific Instrum. 2011, 82:104904, https://doi.org/10.1063/1.3656074

[14] Iwasaki, H.; Yokoyama, S.; Tsukui, T.; Koyano, M.; Hori, H.; Sano, S. Evaluation of the Figure of Merit of Thermoelectric Modules by Harman Method, Jpn. J. Appl. Phys 2003, Vol. 42:3707, https://doi.org/10.1143/JJAP.42.3707

[15] Wang, H.; McCarty, R.; Salvador, J.R.; Yamamoto, A.; König, J. Determination of thermoelectric module efficiency: A survey", Journal of Elec Materi 2014, Vol. 43:2274, doi: 10.1007/s11664-014-3044-2

[16] McCarty, R.; Piper, R. Voltage-Current Curves to Characterize Thermoelectric Generators. Journal of Elec Materi 2015, Vol. 44:1896, doi: 10.1007/s11664-014-3585-4

[17] Min, G.. Principle of determining thermoelectric properties based on $1-V$ curves. Meas. Sci. Technol. 2014, Vol. 25:085009, doi: 10.1088/0957-0233/25/8/085009

[18] Mahajan, S.B.; Pierce, R.D.; Stevens, R.J. Characterizing high temperature thermoelectric modules. In International Mechanical Engineering Congress and Exposition (IMECE), V06AT07A094; 10 pages. ASME, 2013. doi: 10.1115/IMECE2013-66120

[19] Min, G.; Rowe, D. M.; Kontostavlakis, K. Thermoelectric figure-of-merit under large temperature differences. J. Phys. D: Appl. Phys. 2004, 37:1301-1304. 
[20] Zabrocki, K. Continuum Theory of TE Elements. In Continuum Theory and Modeling of Thermoelectric Elements; Goupil, C., Eds.; Wiley-VCH: Berlin, Germany, 2016, pp. 1-74, ISBN: 9783527413379

[21] Kim, H.S.; Liu, W.; Chen, G.; Chu, C.-W., Ren, Z. Relationship between thermoelectric figure of merit and energy conversion efficiency. Proc. Natl. Acad. Sci. USA 2015, 112:8205.

[22] Armstrong, H.; Boese, M.; Carmichael, C.; Dimich, H.; Seay, D.; Sheppard, N.; Beekman, M. Estimating Energy Conversion Efficiency of Thermoelectric Materials: Constant Property Versus Average Property Models. J. of Elec. Matria. 2017, 46:6.

[23] ASTM C177-19. Standard Test Method for Steady-State Heat Flux Measurements and Thermal Transmission Properties by Means of the Guarded-Hot-Plate Apparatus, ASTM International, West Conshohocken, PA, 2019, doi: 10.1520/C0177-19

[24] Anatychuk, L.I.; Havrylyuk, M.V. Procedure and Equipment for Measuring Parameters of Thermoelectric Generator Modules. Journal of Elec Materi 2011, Vol. 40:1292, doi: 10.1007/s11664-011-1619-8

[25] Rauscher, L.; Fujimoto, S.; Kaibe, H.T.; Sano, S. Efficiency determination and general characterization of thermoelectric generators using an absolute measurement of the heat flow. Measurement Science and Technology 2005, Vol. 16:1054, doi: 10.1088/09570233/16/5/002

[26] Kwon, S.; Kim, Y.G.; Lee, S.; Kim, J.C. Measurement of The Figure-of-Merit of Thermoelectric Devices. In XX IMEKO World Congress Metrology for Green Growth, Busan, Republic of Korea, 2012

[27] National Physical Laboratory - https://www.npl.co.uk/products-services/advancedmaterials/thermal-performance - Retrieved: 2019-10-23

[28] Blumm, J.; Lindemann, A.; Niedrig, B. Measurement of the thermophysical properties of an NPL thermal conductivity standard Inconel 600. Proc. of 17th European Conference on Thermophysical Properties, Thessaloniki, Greece 2003, p. 621-626.

[29] BIPM, IEC, IFCC, ILAC, ISO, IUPAC, IUPAP, OIML. Guide to the Expression of Uncertainties in Measurement. Joint Committee for Guides in Metrology 100:2008, GUM 1995 with minor corrections, http://www.bipm.org/utils/common/documents/jcgm/JCGM 1002008 E.pdf, Retrieved: 2019-10-23

[30] Woodside, W. Analysis of Errors Due to Edge Heat Loss in Guarded Hot Plates. Symposium on Thermal Conductivity Measurements and Applications of Thermal Insulations, ASTM STP 1957, 217:49-64.

[31] Donaldson, I. G. A Theory for the Square Guarded Hot Plate-A Solution of the Heat Conduction Equation for a Two Layer System. Quarterly of Applied Mathematics 1961, 19:205-219.

[32] Pratt, A. W. Analysis of Error Due to Edge Heat Loss in Measuring Thermal Conductivity by the Hot Plate Method. Journal of Scientific Instruments 1962, 39:63-68

[33] Troussart, L. R. Three-Dimensional Finite Element Analysis of the Guarded Hot Plate Apparatus and its Computer Implementation. Journal of Thermal Insulation 1981, 4:225-254. 